\title{
Cellular human tissue-engineered skin substitutes investigated for deep and difficult to heal injuries
}

\author{
Álvaro Sierra-Sánchez (iD $^{1,2,6 凶}$, Kevin H. Kim ${ }^{3,4,6}$, Gonzalo Blasco-Morente ${ }^{4}$ and Salvador Arias-Santiago ${ }^{1,2,4,5}$
}

Wound healing is an important function of skin; however, after significant skin injury (burns) or in certain dermatological pathologies (chronic wounds), this important process can be deregulated or lost, resulting in severe complications. To avoid these, studies have focused on developing tissue-engineered skin substitutes (TESSs), which attempt to replace and regenerate the damaged skin. Autologous cultured epithelial substitutes (CESs) constituted of keratinocytes, allogeneic cultured dermal substitutes (CDSs) composed of biomaterials and fibroblasts and autologous composite skin substitutes (CSSs) comprised of biomaterials, keratinocytes and fibroblasts, have been the most studied clinical TESSs, reporting positive results for different pathological conditions. However, researchers' purpose is to develop TESSs that resemble in a better way the human skin and its wound healing process. For this reason, they have also evaluated at preclinical level the incorporation of other human cell types such as melanocytes, Merkel and Langerhans cells, skin stem cells (SSCs), induced pluripotent stem cells (iPSCs) or mesenchymal stem cells (MSCs). Among these, MSCs have been also reported in clinical studies with hopeful results. Future perspectives in the field of human-TESSs are focused on improving in vivo animal models, incorporating immune cells, designing specific niches inside the biomaterials to increase stem cell potential and developing three-dimensional bioprinting strategies, with the final purpose of increasing patient's health care. In this review we summarize the use of different human cell populations for preclinical and clinical TESSs under research, remarking their strengths and limitations and discuss the future perspectives, which could be useful for wound healing purposes.

npj Regenerative Medicine (2021)6:35; https://doi.org/10.1038/s41536-021-00144-0

\section{SKIN WOUND HEALING}

Skin is a vital organ with multitude of functions, one of which is to serve as a barrier to protect against external agents that can cause serious harm. Its relevance becomes apparent with extensive loss of skin due to deep injuries or burns, which affect many parts of human body (limbs, back, and trunk). Delayed intervention can lead to chronic wounds generation or fluid and electrolyte imbalance, poor thermal regulation and sepsis that can ultimately cause death ${ }^{1}$.

To avoid these undesired outcomes, a complex but wellorchestrated process divided in four overlapping phases (hemostasis, inflammation, proliferation, and remodeling) called wound healing (Fig. 1), plays a crucial role after a cutaneous injury, restoring function and appearance of damaged skin with minimal scarring ${ }^{2}$.

This process requires the involvement and coordination of many cell types and signaling pathways ${ }^{3}$. Firstly, vasoconstriction is achieved due to endothelin and factors released by injured cells, such as epinephrine and catecholamines, and moreover, platelets produce platelet-derived growth factor (PDGF), which activates mesenchymal cells from smooth muscles in the vessel walls causing contraction ${ }^{3,4}$. To conclude hemostasis, platelets, through $G$ protein-coupled receptor, bind to thrombogenic subendothelial matrix ${ }^{5}$, activating integrins (allb $\beta 3$ or $\mathrm{a} 2 \beta 1$ ) and glycoproteins (lb$I X-V$ and $V I)$, which increase the attachment to fibrinogen, fibronectin and von Willebrand factor and between platelets (platelets plugs) ${ }^{6,7}$. Finally, platelets within the plug releases many growth factors (PDGF, transforming growth factor $\beta$-TGF- $\beta$-, or epidermal growth factor -EGF-), required for the next stages and moreover, provide a surface for assembly and activation of coagulation complexes lead by Factor $\mathrm{X}$, and where, after Factor XIII crosslinks fibrin, thrombus is formed serving as provisional wound matrix ${ }^{3,8}$.

In inflammatory phase, transcription-independent pathways $\left(\mathrm{Ca}^{2+}\right.$ waves, reactive oxygen species gradients, and pyrogenic molecules) and damaged associated factors such as $\mathrm{H}_{2} \mathrm{O}_{2}$ are responsible of inflammatory cells recruitment, activating keratinocytes regeneration and promoting new vessel formation ${ }^{3,9}$. In particular, neutrophils secrete antimicrobial agents and phagocyte bacteria and cell debris, meanwhile, macrophages have a microbicidal and pro-inflammatory effect at the beginning, but then, develop an anti-inflammatory role, which accelerate wound healing through the formation of new vessel $(\mathrm{Tie} 2+)$ and the release of vascular endothelial growth factor (VEGF) ${ }^{3,10}$. Moreover, they participate in proliferation phase; inducing the transition of dermal fibroblasts into myofibroblasts and depositing collagen and other extracellular matrix (ECM) components, and also in reepithelization and remodeling; releasing proteases and phagocytizing excessive cells and matrix no required ${ }^{3,8,10}$. Mast cells are also important for wound contraction because synthesized enzymes chymase and tryptase, as well as histamines and VEGF, which stimulates keratinocyte proliferation and re-epithelialization and enhances fibroblast proliferation and collagen synthesis ${ }^{3,11}$.

Proliferation phase is also triggered by many different cell types $^{12}$. The most important are endothelial cells, which are responsible of angiogenesis in response to factors such as VEGF,

\footnotetext{
${ }^{1}$ Cell Production and Tissue Engineering Unit, Virgen de las Nieves University Hospital, Andalusian Network of Design and Translation of Advanced Therapies, Granada, Spain. ${ }^{2}$ Biosanitary Institute of Granada (ibs.GRANADA), Granada, Spain. ${ }^{3}$ Department of Dermatology, University of Arkansas for Medical Sciences, Little Rock, AR, USA. ${ }^{4}$ Department of Dermatology, Virgen de las Nieves University Hospital, Granada University, Granada, Spain. ${ }^{5}$ Department of Dermatology, Faculty of Medicine, University of Granada, Granada, Spain. ${ }^{6}$ These authors contributed equally: Álvaro Sierra-Sánchez, Kevin H. Kim. ${ }^{凶}$ email: alvarosisan@gmail.com
} 


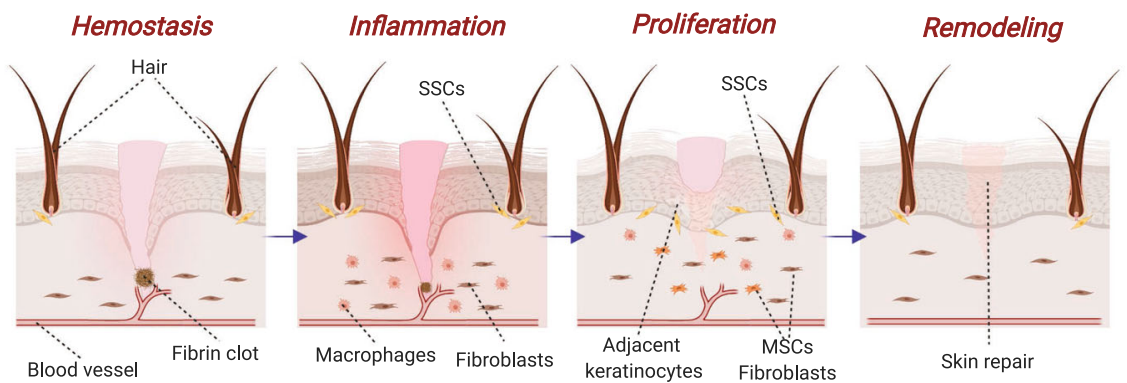

Fig. 1 Phases of skin wound healing process. Hemostasis: activation of fibrin is responsible of clot formation and bleeding is stopped. Inflammation: damaged cells are phagocyted and factors are released to provoke cell migration and proliferation. Proliferation: cells such as dermal fibroblasts, MSCs and SSCs (mesenchymal and skin stem cells) achieve wound's site and form a provisional extracellular matrix. Remodeling: collagen fibers are realigned, and residues are removed. Created with BioRender.com.

PDGF, TGF- $\beta$, and fibroblast growth factor (FGF). This is regulated by Notch pathways through VEGF-A produced by subcutaneous adipose stromal cells $s^{3,13}$. In addition, fibroblasts synthesize ECM and express genes that are responsible of its proliferation and migration, and myofibroblasts, transient cells derived from local fibroblasts and others cells such as mesenchymal stem cells and epithelial cells, also deposit ECM and exhibit contractile characteristics; processes that are fundamental for wound healing ${ }^{3}$.

Finally, regeneration of the dermis is favorable due to their fibrous nature, allowing for migration and proliferation of macrophages and fibroblasts necessary for remodeling and promoting connective tissue formation ${ }^{3,10}$. In the case of epidermal layer, re-epithelialization is a complicated process where keratinocytes located in the wound edge loss their adhesions and express integrins, which leads to increased ErkMAPK signaling and inflammatory cytokine synthesis, causing hyperproliferation of keratinocytes and immune cell activation ${ }^{3,14}$. In addition, human skin stem cells (hSSCs) migrate from their niches in order to replace the lost keratinocytes $8,12,15$ and also express higher levels of integrins ( $\alpha 2 \beta 1, \alpha 3 \beta 1, a 6 \beta 4)$, that binds collagen or laiminin ${ }^{3}$, and release growth factors, which participate in generation of epithelial cells like keratinocytes, promoting re-epithelization of injured skin $^{12,16-18}$.

On balance, wound healing is a coordinated and complex process where many factors such as, inflammatory skin diseases, deep injuries, large sized or chronic wounds can provoke a deregulation due to an altered immune response and the lack of local adult skin cells and hSSCs available for migration, which causes problems to achieve a correct homeostatic restoration ${ }^{15,19,20}$.

When these critical cells are lacking due to deep and difficult to heal wounds, human mesenchymal stem cells (hMSCs) can also contribute to re-epithelization ${ }^{21}$ by stimulating collagen production and reducing fibrosis and scar formation by releasing many growth factors such as EGF or basic fibroblast growth factor $(\mathrm{bFGF})^{12}$.

Hence, much of the efforts have been dedicated to understanding the mechanisms of wound healing and to develop clinically viable therapies based on tissue engineering, to help patients restore function of damaged skin.

\section{TISSUE ENGINEERING AND MATERIAL SCIENCE OF SKIN}

Tissue engineering (TE) is an interesting and growing multidisciplinary field that involves several biomedical areas such as cell biology, material science, engineering or medicine. It appears as a necessity to solve the lack of organ donors or another efficient substitute for the organ required. For this reason, TE tries to manufacture artificial organs and tissues under controlled conditions to be transplanted in vivo in those cases where own patient's regenerative or reparative capacities are not achieved ${ }^{22}$.
Study of TE strategies requires to evaluate many aspects such as cell sources, cell nature, material science, incorporation or not of growth factors and disease models required (injuries and animals). Regarding cell biology, selection of an appropriate cell type will depend on the target tissue but the main challenge for clinical use will be to select among allogeneic (stem cells included) or autologous source due to the advantages and disadvantages associated to each one ${ }^{22-24}$. The other important aspect in TE is material science; first approaches were based on the use of synthetic biomaterials that provided structural support and replaced organs but without functionality ${ }^{23}$. However, research of ECM has provoked the development of new biomaterials capable of resembling biological and mechanical aspects such as three-dimensional (3D) structures (scaffolds), which enable nutrient's transport and vascularization ${ }^{22-24}$. Considering their nature, biomaterials could be synthetic, naturally derived or acellular tissue matrices and they must be biocompatible, biodegradable and bioresorbable to be replaced by native tissue without rejection ${ }^{23}$.

In the case of skin, a tissue-engineered skin substitute (TESS) is any safe product, constituted of human cells and bio-scaffolds, capable of replacing damaged human skin and resembling its structural and functional characteristics such as flexibility, protective barrier or transepidermal water loss ${ }^{25,26}$.

In most TESSs, the cellular component is composed of human adult keratinocytes and fibroblasts as part of epidermal and dermal layers, respectively ${ }^{20}$. However, due to the many advantageous properties ${ }^{8,27}$ of human stem cells (hSCs) and the specific role of hSSCs and hMSCs in restoring homeostatic conditions $^{12,15}$, new approaches in the field of skin engineering are focusing on the incorporation of these cell types to TESSs ${ }^{21}$ (Table 1).

Several biomaterials such as collagen ${ }^{28}$, chitosan ${ }^{29}$, elastin ${ }^{30}$, or hyaluronic acid ${ }^{31,32}$ have been used for manufacturing TESSs. On balance, they differ in their internal structure: porous, fibrous, hydrogel, or ECM nature, which provide advantageous and drawbacks depending on therapeutic purposes ${ }^{33}$.

In this review, we analyze the different cell types, human adult skin cells but also human stem cells, used to develop research models of TESSs, at preclinical or clinical environment, for the treatment of deep and difficult to heal wounds.

\section{HUMAN ADULT SKIN CELLS IN TESSS}

Human skin is composed of several cell types distributed in the different layers of skin: epidermis, dermis and hypodermis. Epidermis is mainly composed of keratinocytes, but also melanocytes, Langerhans cells and Merkel cells are present. Dermis is primarily constituted by fibroblasts and extracellular matrix, meanwhile, hypodermis is mainly comprised of adipose tissue cells. 


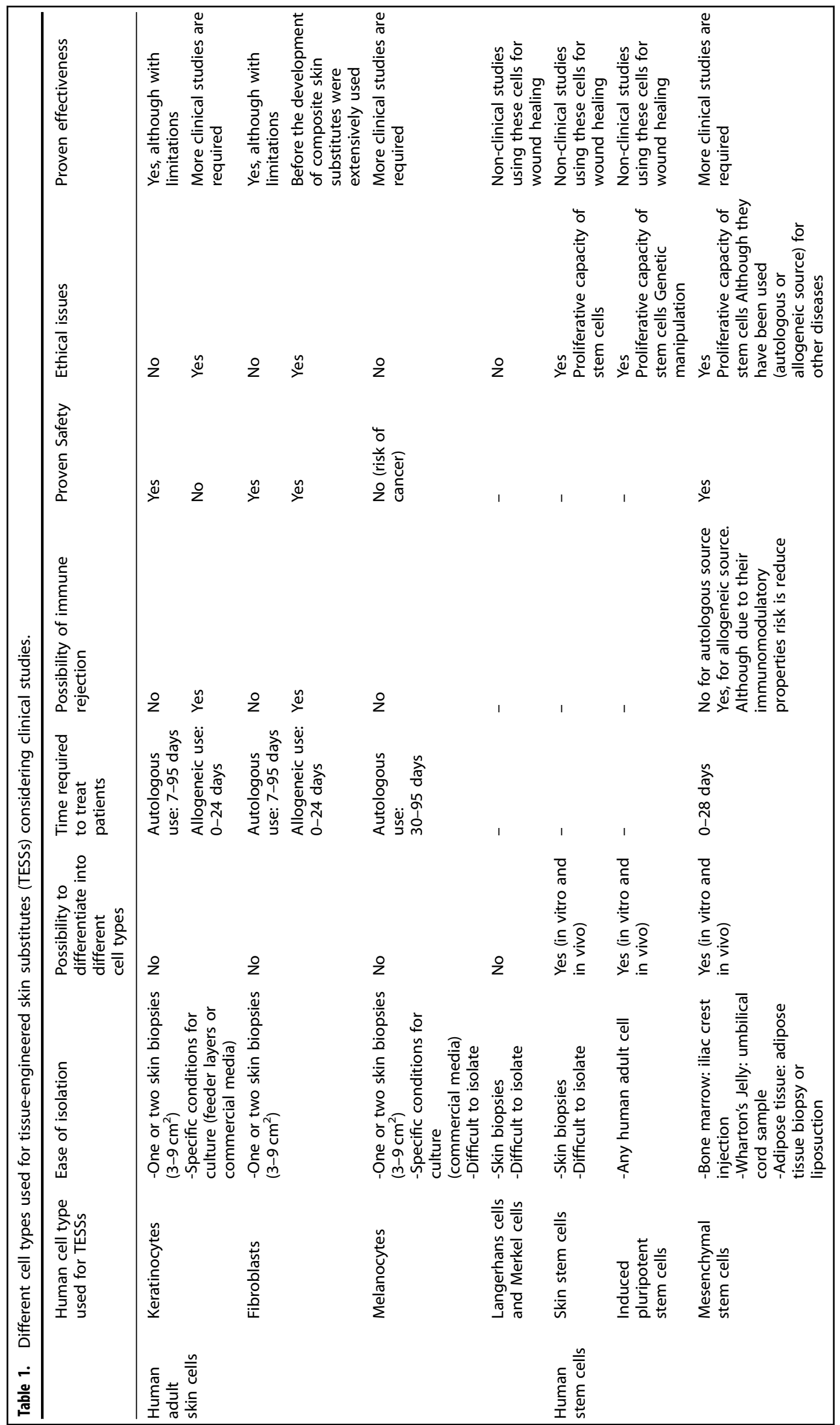


Isolation and expansion of human adult skin cells

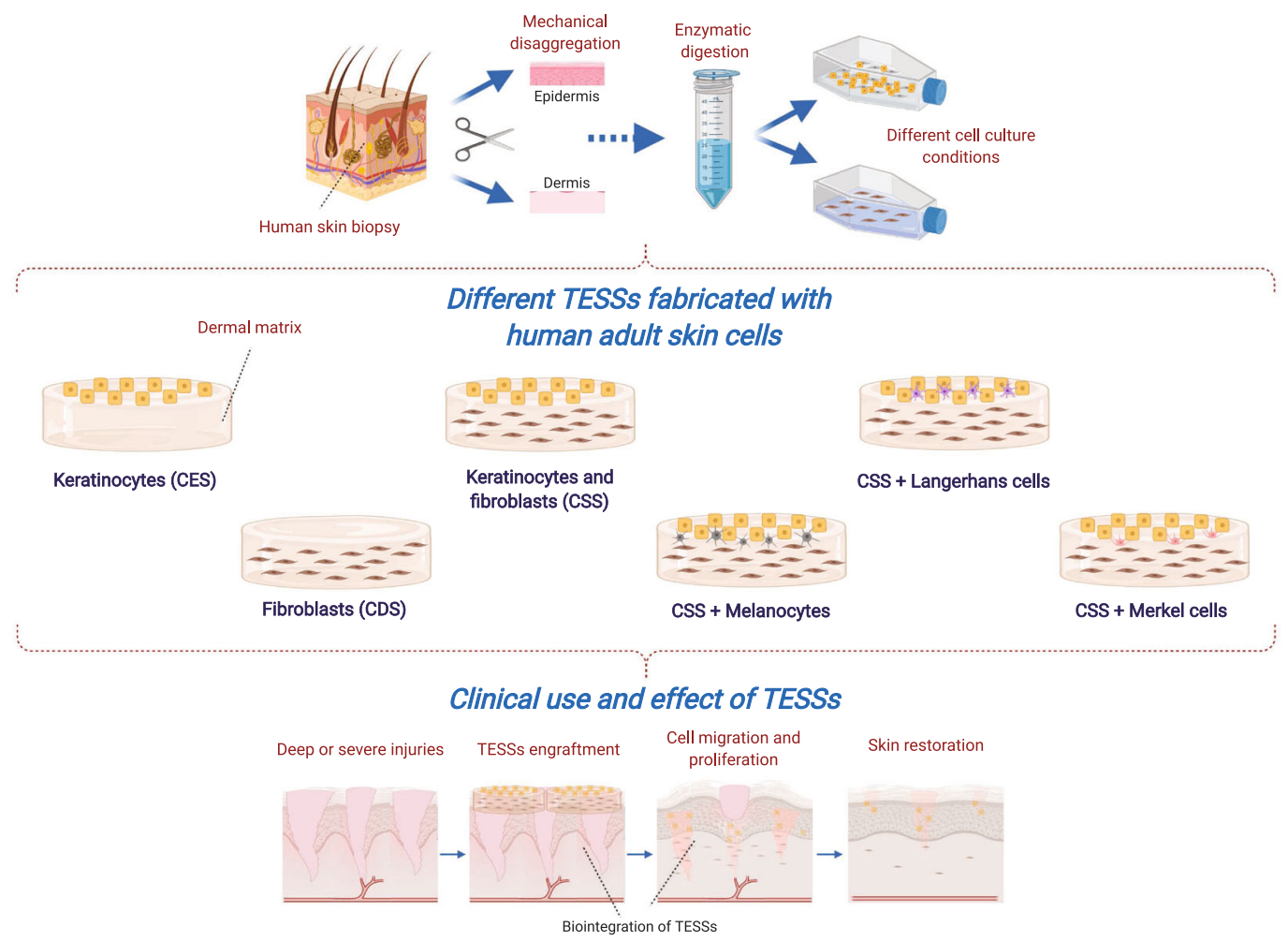

Fig. 2 Tissue-engineered skin substitutes (TESSs) fabricated with human adult skin cells and their role in wound healing process. After a deep, severe or chronic injury where, normal phases of healing are not possible, fabrication of TESSs from cells of a human skin biopsy is the most usual advanced therapy. Keratinocytes, fibroblasts and the rest of epithelial cells are isolated, expanded and used in combination with a biological matrix to produce sheets of cultured epithelial substitutes (CESs), cultured dermal substitutes (CDSs) and composite skin substitutes (CSSs), which are engrafted to promote and facilitate cell activation and the release of growth factors necessary to achieve reparation, regeneration and homeostasis of skin. Created with BioRender.com.

Most of the non-commercial substitutes studied are constituted by epidermal, dermal or both layers, where keratinocytes and fibroblasts are the most used cell types; however, some researchers explored the use of the other epithelial cell types with the purpose of fabricating TESSs that better resemble native skin (Fig. 2).

\section{Human keratinocytes for preclinical TESSs}

Keratinocytes were the first skin cell type isolated and explored ${ }^{34}$, and for this reason the former models of TESSs were based on these cells only. Development of cultured epithelial substitutes (CESs) for burn patients has been one of the main objectives, which has led to many extensively studied commercial devices ${ }^{35,36}$.

In recent years, the number of studies evaluating the use of human keratinocytes-only TESSs have been limited. Some authors have used these CESs to compare different culture techniques ${ }^{37}$ or dermal matrices (ECM derived from fibroblasts) ${ }^{38}$. The necessity of including dermal components to support in vivo proliferation and preservation of keratinocytes was early demonstrated in athymic mice by Rennekampff et al. $^{39}$ where human keratinocytes transplanted with an acellular dermal matrix onto full-thickness skin defects, developed a fully differentiated epidermis and persisted in all animals grafted (vs. $63.6 \%$ of those animals without a dermal component).

In 2019, Horch et al. ${ }^{40}$ studied in vivo the use of keratinocyte monolayers in hyaluronic acid membranes demonstrating that when keratinocytes were directly implanted towards the fullthickness wound bed of athymic mice, formation of a multilayered and differentiating epidermis was faster (14 days) than conventional technique ( $>21$ days).

\section{Human keratinocytes for clinical TESSs}

Owing to the important role of epidermis as a protective barrier, CESs with keratinocytes were the first TESSs explored in patients (Table 2). Since 1981, many clinical studies have analyzed the role of CESs in skin regeneration ${ }^{41-56}$, mainly for the treatment of burns (14 of 16 studies), although treatment of surgical wounds was also reported.

Regarding to culture and manufacturing process of CESs, in most of former studies reviewed (in chronological order), keratinocytes were expanded and isolated by enzymatic detachment from culture flasks and directly engrafted onto patients ${ }^{41-}$ $48,50,52,54$. In these cases, results differed depending on: parameters analyzed, endpoint of follow-up or pretreatment strategy, but, in general, studies that only applied this type of CES reported worse results in terms of graft take, due probably to the effect of digestive enzymes on epidermal cells.

For this reason, some authors concluded that their use could be interested as temporary biological dressing ${ }^{54}$ or combined with meshed split-thickness skin grafting ${ }^{42,43,48}$. In other cases, the previous engraftment of artificial $^{52}$ or allogeneic split-thickness skin grafts ${ }^{43,46}$ improved the take of these conventional CESs and accelerate wound healing ${ }^{52}$ due to the increase of capillarity density $^{43}$.

However, due to the risk associated with these strategies, researches started to investigate the fabrication of more complex CESs where epithelial cells and biomaterials were cultured in vitro 


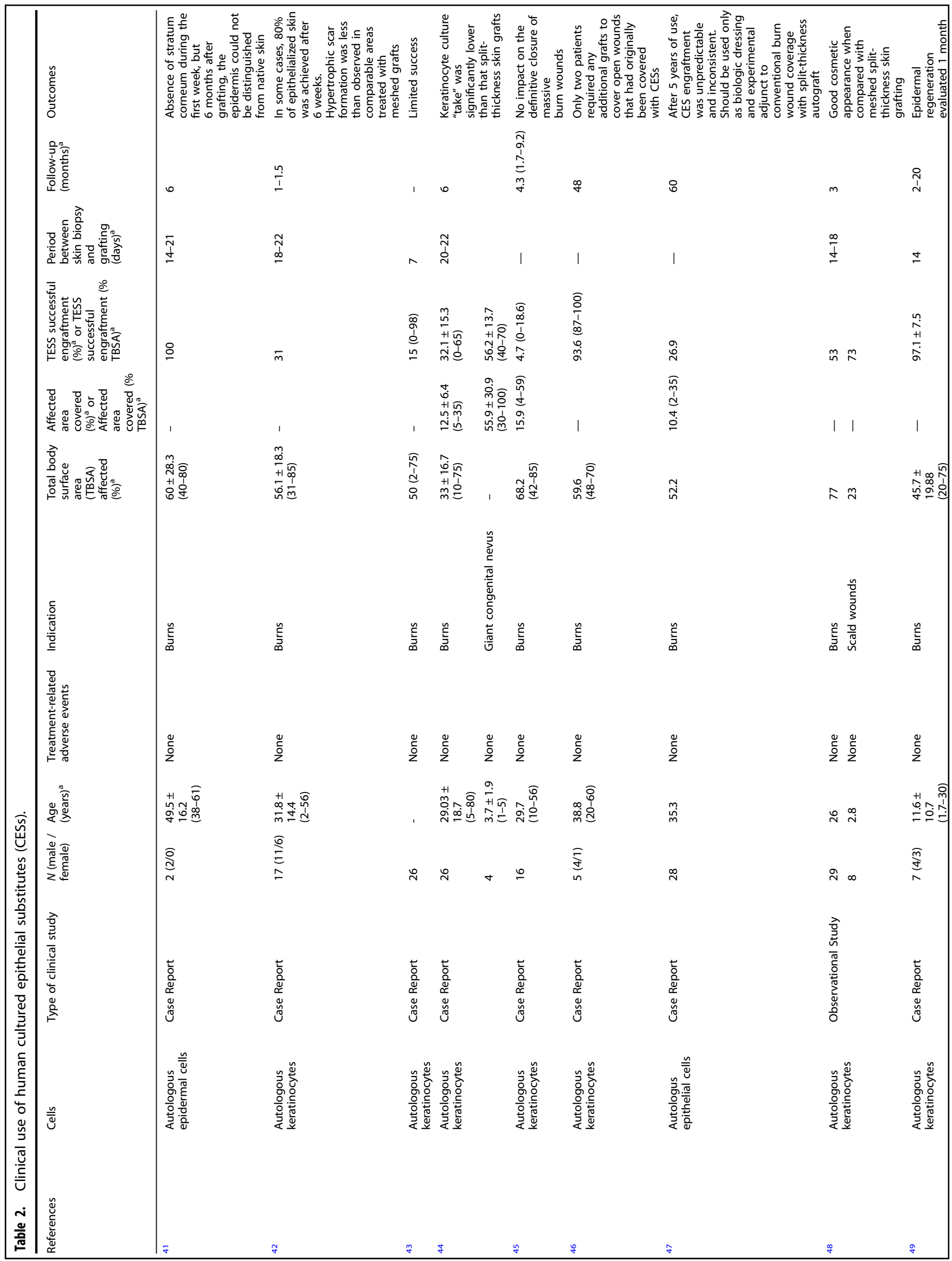




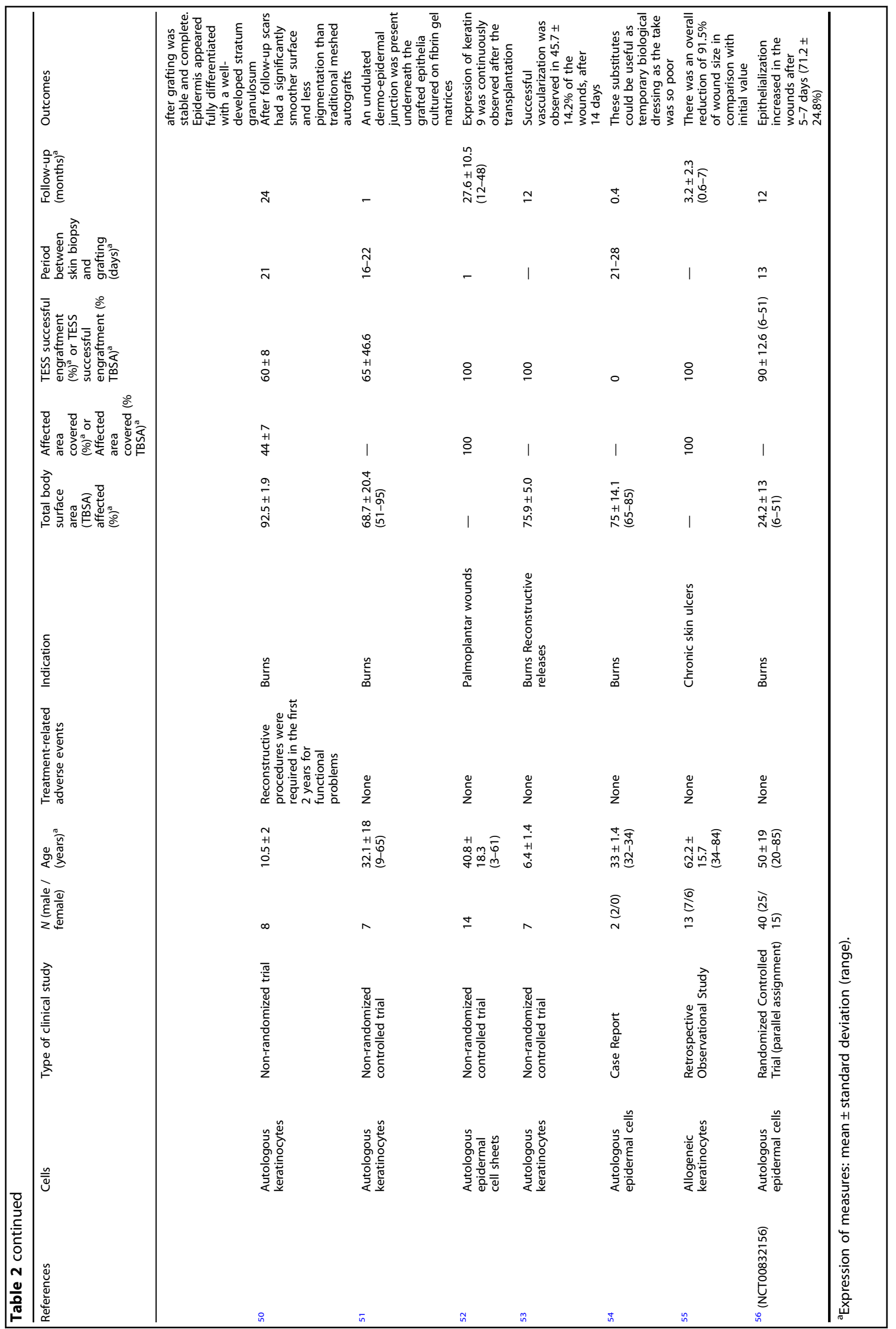


before engraftment ${ }^{49,51,53,55,56}$. Two studies evaluated the use of fibrin and in both cases take of grafts was higher, improving the relation cost-efficiency ${ }^{49}$ and demonstrating that fibrin facilitated the formation of dermo-epidermal junction because ECM proteins secreted by autologous keratinocytes were retained ${ }^{51}$.

Sheridan et al. ${ }^{53}$ and Pajardi et al..$^{55}$ developed CESs based on acellular dermis or membranes, respectively. In the first case $\mathrm{e}^{53}$, vascularization after 14 days was higher in the case of autografts ( $98 \pm 1 \%$ vs. $45.7 \pm 14.2 \%$ ), however, results of Vancouver Scar Scores (VSSs) after 12 months demonstrated that no differences existed between autologous CESs (1.2 \pm 0.7$)$ and autografts ( $1.0 \pm$ 0.4). In the second case ${ }^{55}$, at the end of the follow-up, a reduction of $91.5 \%$ of wound dimensions was achieved with allogeneic CESs.

Other biomaterials used were collagen and elastin ${ }^{56}$ but, in this case, autologous CESs were engrafted before gold standard treatment (autografts) was applied. Results revealed that the combination of CESs and autografts increased epithelization against those cases where only autografts were applied (71\% vs. $67 \%$ ), and after 12 months of follow-up, Patient and Observer Scar Assessment Scale (POSAS) reported better results when CESs were grafted (14.2 \pm 7.2 vs. $18.4 \pm 10.2)$. This was the only randomized controlled clinical trial reviewed (NCT00832156) ${ }^{56}$.

Interestingly, one of the studies demonstrated the importance of using an appropriate skin autograft or source. Yamaguchi et al. $^{52}$ compared three different treatments for palmoplantar wounds: (i) CESs with autologous epidermal cells from palmoplantar sites, (ii) no-palmoplantar skin grafts, and (iii) palmoplantar skin grafts. No expression of keratin 9 was observed in the case of no-palmoplantar skin grafts and after 1 year, wound size was higher $\left(26.77 \pm 6.72 \mathrm{~cm}^{2}\right)$ than in those patients treated with CESs $\left(12.27 \pm 4.14 \mathrm{~cm}^{2}\right)$ and palmoplantar skin grafts $\left(4.24 \pm 0.68 \mathrm{~cm}^{2}\right)$.

On balance, studies using CESs have evolved from the first reported, including new culture techniques and strategies, however, it does not seem to be the best alternative when deep wounds or difficult to heal wounds needs to be treated. To date, a total of 259 patients $(29.1 \pm 17.2$ years old), the majority of them with burn injuries (88.0\% of the cases), with a mean of $57.4 \pm$ $20.0 \%$ total body surface area (TBSA) affected, have been treated using this strategy, and the percentage of successful engraftment was $60.5 \pm 35.0 \%$ without adverse events except in one case, where more reconstructive procedures were required for functional problems ${ }^{50}$ (Table 2).

\section{Human fibroblasts for preclinical TESSs}

Development of human cultured dermal substitutes (CDSs) is essential to achieve a proper integration of the engraftment and successful wound healing. Mineo et al. $^{31}$ developed a dermal substitute composed of hyaluronic acid, collagen and human dermal fibroblasts. They demonstrated in vitro, increased amount of VEGF and hepatocyte growth factor (HGF), which effectively created a vascularized wound bed for autologous skin grafting in Sprague Dawley rats with deep dermal burns.

Other studies explored the role of extracellular matrix of human fibroblasts to support the growth of these cells and develop more natural substitutes ${ }^{57,58}$ for full-thickness wounds, demonstrating in vitro and in vivo, on Sprague Dawley rats, that they have notable effects on wound healing, facilitating fibroblast infiltration, collagen bundle production, and elastic fiber and blood vessel formation ${ }^{58}$.

Mohd Hilmi et al. ${ }^{29}$ evaluated a chitosan sponge matrix seeded with human dermal fibroblasts, engrafted onto full-thicknesses wounds excised on the irradiated skin of Sprague Dawley rats. Wounds treated with chitosan CDS showed the most reepithelialization level $(33.2 \pm 2.8 \%)$ and scar size of wounds were significantly decreased compared with control group where duoderm CGF was applied $(0.13 \pm 0.02 \mathrm{~cm}$ vs. $0.45 \pm 0.11 \mathrm{~cm})$.
Finally, the addition of other cell types such as endothelial cells could be useful to increase the regeneration potential of CDSs. One study fabricated a TESS based on endogenous matrix produced by human dermal fibroblasts and cultured with human fibroblasts and endothelial cells, which were capable of forming capillary-like-structures effectively anastomosed with host vessels in vivo ${ }^{59}$.

\section{Human fibroblasts for clinical TESSs}

With the advancement of culture techniques and ability to isolate dermal fibroblasts, clinical studies have evaluated the use of CDSs for the treatment of chronic skin ulcers (7 of 10 studies), surgical wounds and burns (Table 3$)^{55,60-68}$. Important findings from these studies highlight the release of cytokines or growth factors, which activates many pathways for skin regeneration ${ }^{60,63}$.

In most of the cases, CDSs were fabricated using allogeneic fibroblasts ${ }^{55,60-65,68}$. Cells were cultured over different scaffolds and placed cell-seeded side down onto the wound surface.

Regarding to the type of clinical studies reviewed, three were considered as clinical trials ${ }^{66-68}$, one was an observational study ${ }^{55}$ and remaining were classified as case reports ${ }^{60-65}$.

Among these, three studies compared different therapies ${ }^{63,66,68}$. Yamada et al. ${ }^{63}$ evaluated the use of allogeneic fresh or cryopreserved fibroblasts cultured on a bilayer sponge composed of hyaluronic acid and collagen for the treatment of deep surgical wounds, demonstrating that cryopreserved cells were capable of releasing cytokines and promoting re-epithelialization at the same level as fresh cells. You et al. ${ }^{66}$ reported better results in terms of complete ulcer healing when compared the use of hyaluronic acid-based autologous CDSs (84\%) and non-adherent foam dressings (34\%). Finally, Momeni et al. ${ }^{68}$ studied the use of amniotic membranes alone or combined with allogeneic fibroblasts and compared the results with a control therapy (Vaseline gauze). Results revealed that wound closure of surgical wounds was higher when amniotic membranes were used (alone- $95.5 \%$ -, with fibroblasts-94\%-vs. control-59\%-) and reepithelialization was faster (alone- $-11.3 \pm 2.9$ days-, with fibroblasts $-10.1 \pm 2.4$ days - vs. control $-14.8 \pm 1.6$ days-).

Interestingly, a combination of hyaluronic acid and collagen was the preferred matrix for the manufacture of CDSs ${ }^{60-65}$. Different wounds were treated using these allogeneic CDSs (burns ${ }^{60}$, surgical wounds ${ }^{63}$, and ulcers ${ }^{61,62,64,65}$ ), but similar results indicated that their application would be interested as biological wound dressing to produce granulation tissue and secrete VEGF, bFGF, and ECM proteins useful for mesh-auto skin grafts.

Finally, Morimoto et al. $^{67}$ used an artificial dermis and autologous fibroblasts for the treatment of diabetic ulcers demonstrating an important wound size reduction after 21 days (from $7.1 \pm 4.9 \mathrm{~cm}^{2}$ to $3.4 \pm 2.3 \mathrm{~cm}^{2}$ ). In contrast, Pajardi et al. ${ }^{55}$ used hyaluronan as dermal matrix for the treatment of chronic ulcers reporting a wound size reduction of $73 \%$.

One-hundred five patients (63 males and 42 females), older than those treated with CESs (58.4 \pm 14.7 years old), were treated with CDSs. Small injuries (87.6\% of cases were chronic skin ulcers) were evaluated and successful engraftment was achieved in $79.5 \pm 20.0 \%$ of the cases. Adverse events were only observed in five cases, related to local infections (Table 3 ).

\section{Combination of human keratinocytes and fibroblasts for preclinical TESSs}

In recent years, the combination of human keratinocytes and fibroblasts in TESSs, called composite skin substitutes (CSSs) has been explored.

CSSs resemble normal skin by containing an epidermal layer of autologous or allogeneic keratinocytes and a dermal layer of fibroblasts incorporated into a stromal scaffold. They not only provide structural dermo-epidermal support, but also deliver 


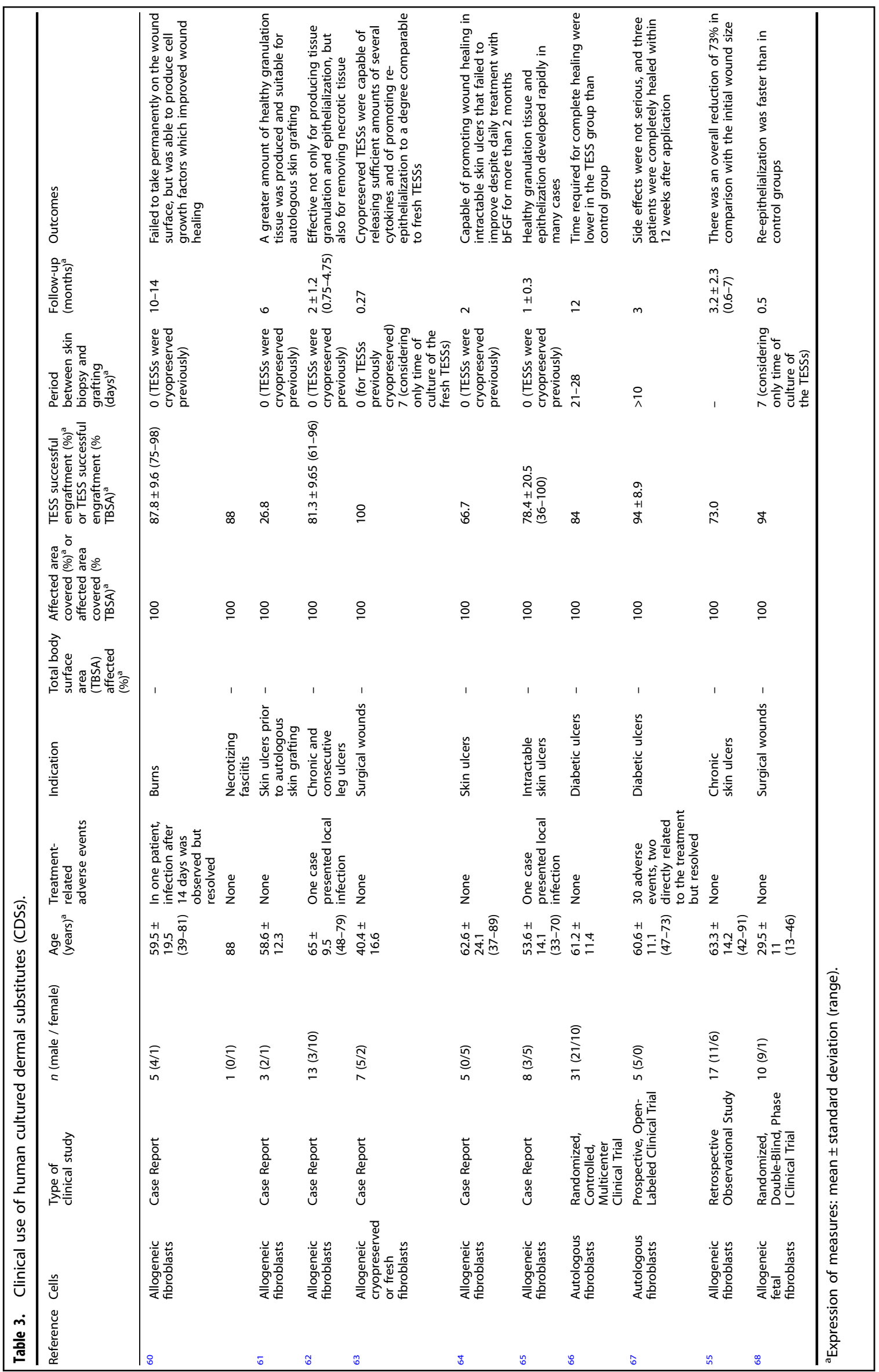


growth factors (EGF, PDGF, VEGF) and extracellular matrix that increase the rates of recovery and healing ${ }^{69,70}$.

For these reasons, many studies have looked different strategies to explore potential benefits of CSSs. A composite TESS manufactured with fibrin-hyaluronic acid biomaterial has been recently evaluated in vivo in immunodeficient mice with excisional wounds and compared with another fibrin-agarose CSS and secondary wound healing dressings, demonstrating favorable outcomes, similar to autografts in terms of clinical (POSAS scale results: eight for CSS vs. six for autografts), homeostasis (transepidermal water loss: $6.42 \pm 0.75 \mathrm{~g} / \mathrm{h} / \mathrm{m}^{2}$ for CSS vs. $6.91 \pm$ $1.28 \mathrm{~g} / \mathrm{h} / \mathrm{m}^{2}$ for autografts) and histological restoration, after eight weeks of engraftment ${ }^{32}$.

Similarly, Tissue Biology Research Unit of Zurich developed human CSSs based on type I collagen hydrogels, demonstrating, in vitro and in vivo in full-thickness skin defects of athymic rats, that these TESSs homogeneously developed a well-stratified epidermis over the entire surface of the grafts and displayed a well-defined basal cell layer where keratin 19/keratin 15-doublepositive keratinocytes are essential in growing $\operatorname{skin}^{71,72}$.

Supp et al. ${ }^{73}$ manufactured composite TESSs based on collagenglycosaminoglycan for studying recessive dystrophic epidermolysis bullosa (RDEB) on immunodeficient mice and demonstrated that formation of structurally normal anchoring fibrils appears to require expression of type VII collagen in both skin layers. Bacakova et al. ${ }^{74}$ also developed collagen-based CSSs by utilizing a nanofibrous poly-L-lactide and observed cell migration and proliferation after 14 days of in vitro culture.

Interestingly, Centre de recherche en organogénèse expérimentale de I'Université Laval/LOEX developed a self-assembly approach, which allows for the production of a scaffold-free cellbased $\mathrm{CSS}^{75,76}$. Briefly, the dermal layer is composed of stacked fibroblast sheets and keratinocytes are seeded onto the tissue, forming a stratified and cornified epidermis. Auger and Germain's group have optimized this protocol and studied this model in vitro and in vivo (athymic mice with full-thickness skin injuries), demonstrating timely production of CSSs that could improve clinical availability for the effective wound coverage of patients ${ }^{77-}$ 80

In addition, these types of TESSs have been used as a research tool to investigate other pathological conditions and learn more about the role of these treatments in wound healing. For instance, bin Busra et al. ${ }^{81}$ demonstrated in vivo in mice, that fibrin-based CSSs enhanced healing of irradiated wounds after radiotherapy, with higher expression of TGF- $\beta 1$, PDGF and VEGF than monolayer substitutes.

The vascularization of CSSs has also been studied by incorporating human endothelial cells ${ }^{82-85}$, with reports showing improvement of graft survival and the formation of vascular networks, which physically resemble normal wound healing process.

\section{Combination of human keratinocytes and fibroblasts for clinical TESSs}

Clinical benefits of CESs and CDSs have been observed in many patients; however, the most studied TESSs have been CSSs composed of human keratinocytes and fibroblasts that have been used for the treatment of several dermatological pathologies since 1989 (Table 4) $^{86-106}$.

Most of the studies evaluated the use of CSSs for the treatment of burns, however, experimental designs differed from cell populations used (autologous [19] $]^{86-89,91,92,94-106}$ vs. allogeneic $\left.[2]^{90,93}\right)$, biomaterials selected, randomization or not, comparison or not with other treatments or pretreatment required.

In those cases where different treatments were compared, engraftment of autografts was the gold standard treatment used as reference for each patient ${ }^{88,90,92,93,95,101,102,104-106}$. In one of the cases where allogeneic cells were used, CSSs did not take and the engraftment of more autografts was required ${ }^{93}$. Rest of comparative studies, reported positive results for CSSs in terms of percentage of TBSA closed ([20.5 $\pm 2.5 \%$ for CSSs vs. $52.1 \pm 2 \%$ for autografts $\left.{ }^{95}\right],[29.9 \pm 3.3 \%$ for CSSs vs. $47.0 \pm 2 \%$ for autografts $^{104}$ ] after 28 days in both cases), time of healing (7.4 \pm 0.9 days for CSSs vs. $7.9 \pm 1.5$ days for autografts ${ }^{90}$ ), appearance (scars were less raised than autografts ${ }^{88}$ ), percentage of wound area closed (95.4\% for CSSs vs. $99 \%$ for autografts after 28 days ${ }^{92}$ ), manipulation (easy in comparison with $\mathrm{CESs}^{102}$ ), protein expression (keratin 19 and type IV collagen ${ }^{105}$ ) or percentage of epithelialization $\left(63.5 \pm 35 \%\right.$ after 21 days of engraftment $\left.{ }^{106}\right)$.

Remaining studies indicated the beneficial role of using CSSs alone ${ }^{89,96,98-100,103}$ or combined with autografts $86,87,91,94,97,101$ for the treatment of deep and difficult to heal injuries, evaluating different parameters such as graft take, histological appearance of new skin and cosmetic and functional outcomes.

Interestingly, some researches remarked the importance, as in the case of CESs, of a pretreatment with auto-dermis or allodermis to increase clinical benefits of $\mathrm{CSSs}^{86,91,94,98,106}$.

Regarding to the biomaterials used for the fabrication of the scaffolds, different types of collagen $87,90,93,95,101,106$ or combined with glycosaminoglycan ${ }^{86,88,89,91,92,104}$ were the preferred sources, although different formulations of plasma/fibrin were also reported $^{94,96,98,102,103}$. Finally, hyaluronic acid ${ }^{97}$ or acellular dermal matrices derived from human fibroblasts ${ }^{99,100,105}$ were evaluated too.

To date, a total of 241 patients ( $25.6 \pm 14.9$ years old) with severe burns $(81.7 \%$ of the cases), surgical wounds or skin ulcers with a mean of $69.2 \pm 11.1 \%$ of TBSA affected have benefited from CSSs with a mean percentage of successful engraftment of $80.2 \pm$ $26.3 \%(0 \%-100 \%)$ with slight adverse events such as local inflammation or increased incidence of exudates (Table 4). Interestingly, eleven of twenty one studies included children and five studying children exclusively ${ }^{91,92,102,104,106}$.

\section{Human melanocytes for preclinical TESSs}

In order to develop a TESS that most resemble natural skin, the incoporation of other cell types present in epidermal layer, such as melanocytes, has been evaluated in preclinical stages.

Liu et al. ${ }^{107}$ were one of the first groups to develop a TESSs composed of human fibroblasts, melanocytes and keratinocyes in a type I collagen gel. In vitro and in vivo results revealed proper integration, morphology and successful repair of skin defects in athymic mice and black skins were observed by 6 weeks after grafting.

Biedermann et al. ${ }^{108,109}$ also developed a pigmented skin composite based on collagen, which was transplanted onto fullthickness skin wounds in rats. After 3 weeks, blood vessels, but no nerve fibers or lymphatic vessels were observed ${ }^{108}$. However, peripheral host nerve fibers were found 15 weeks after transplantation $^{109}$. The same group studied the inflammatory response of these pigmented substitutes, which revealed that granulocytes infiltrate the entire graft at 1 week post-transplantation, while monocyte/macrophage recruitment was observed at 3-12 weeks ${ }^{110}$.

Boyce's laboratory also evaluated the use of human melanocytes in collagen-based TESSs. They analyzed in vitro and in vivo (athymic mice) the incorporation of different densities of cryopreserved and recovered human melanocytes in a human CSS. Melanocytes were localized into the dermal-epidermal junction of skin substitutes and were capable of restoring cutaneous pigmentation and ultraviolet photoprotection after full-thickness skin loss conditions ${ }^{111}$, which was corroborated by Goyer et al. ${ }^{112}$, regardless of whether light or dark pigmentation phototype melanocytes were used ${ }^{113}$. 


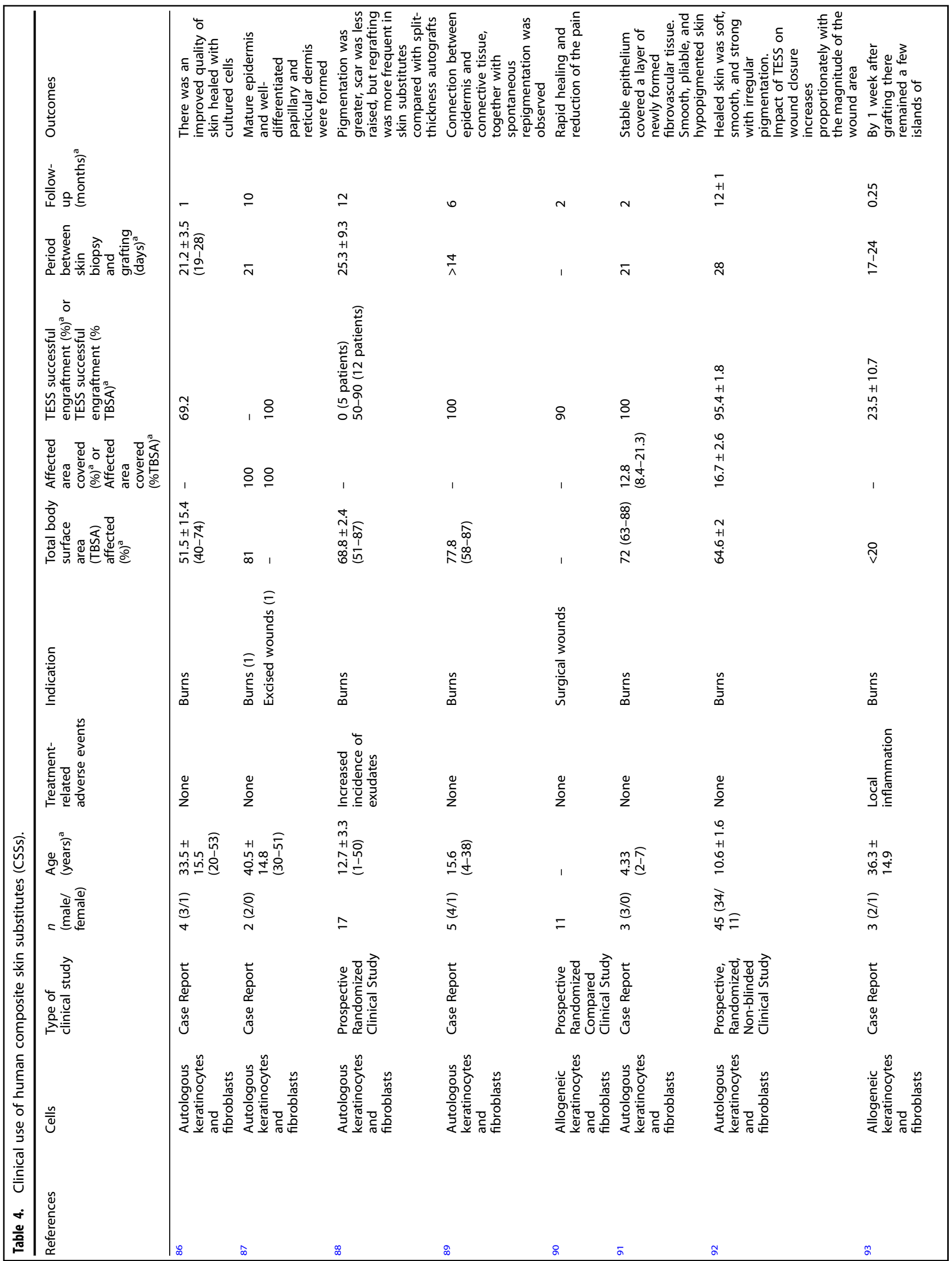




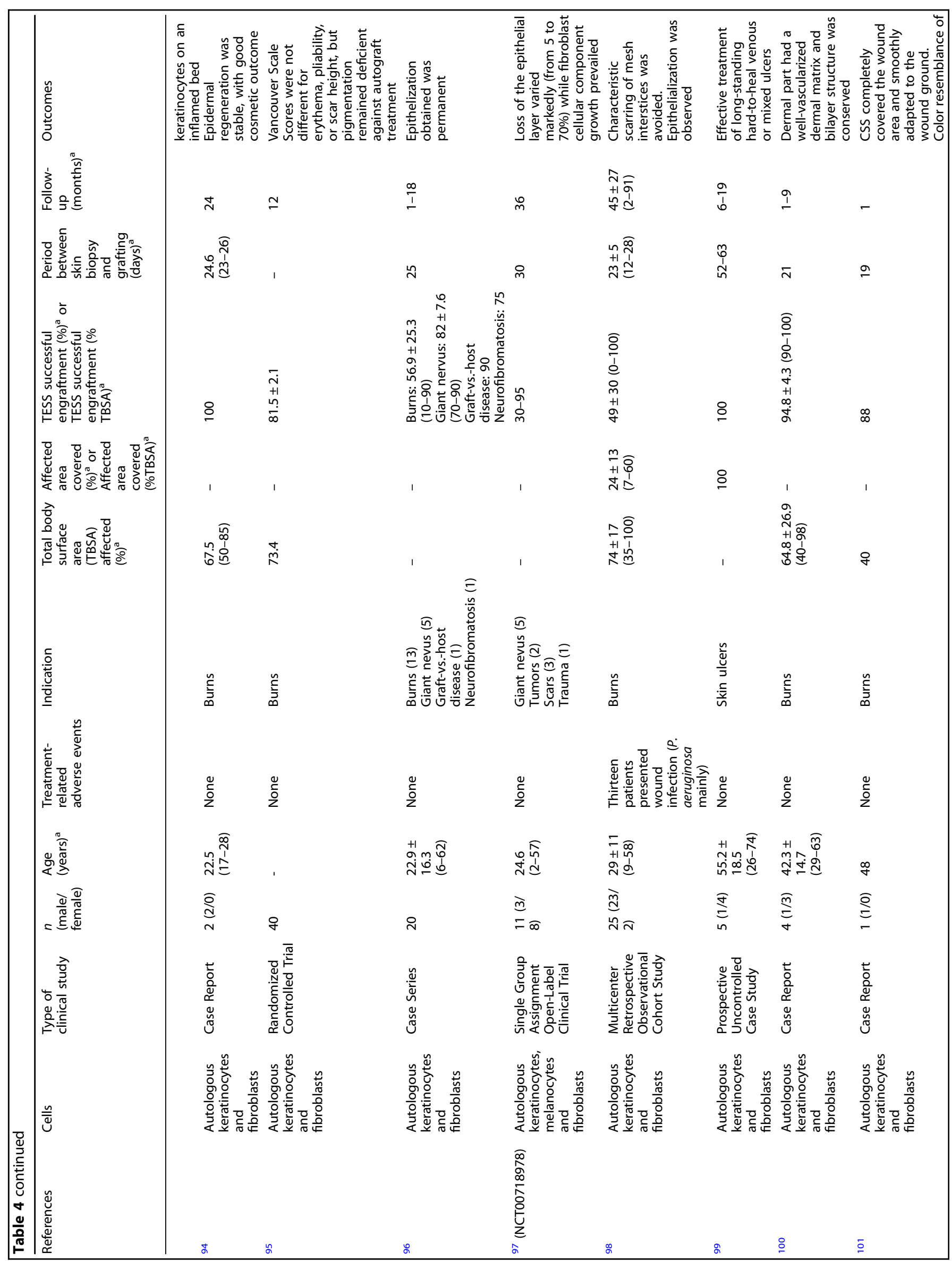




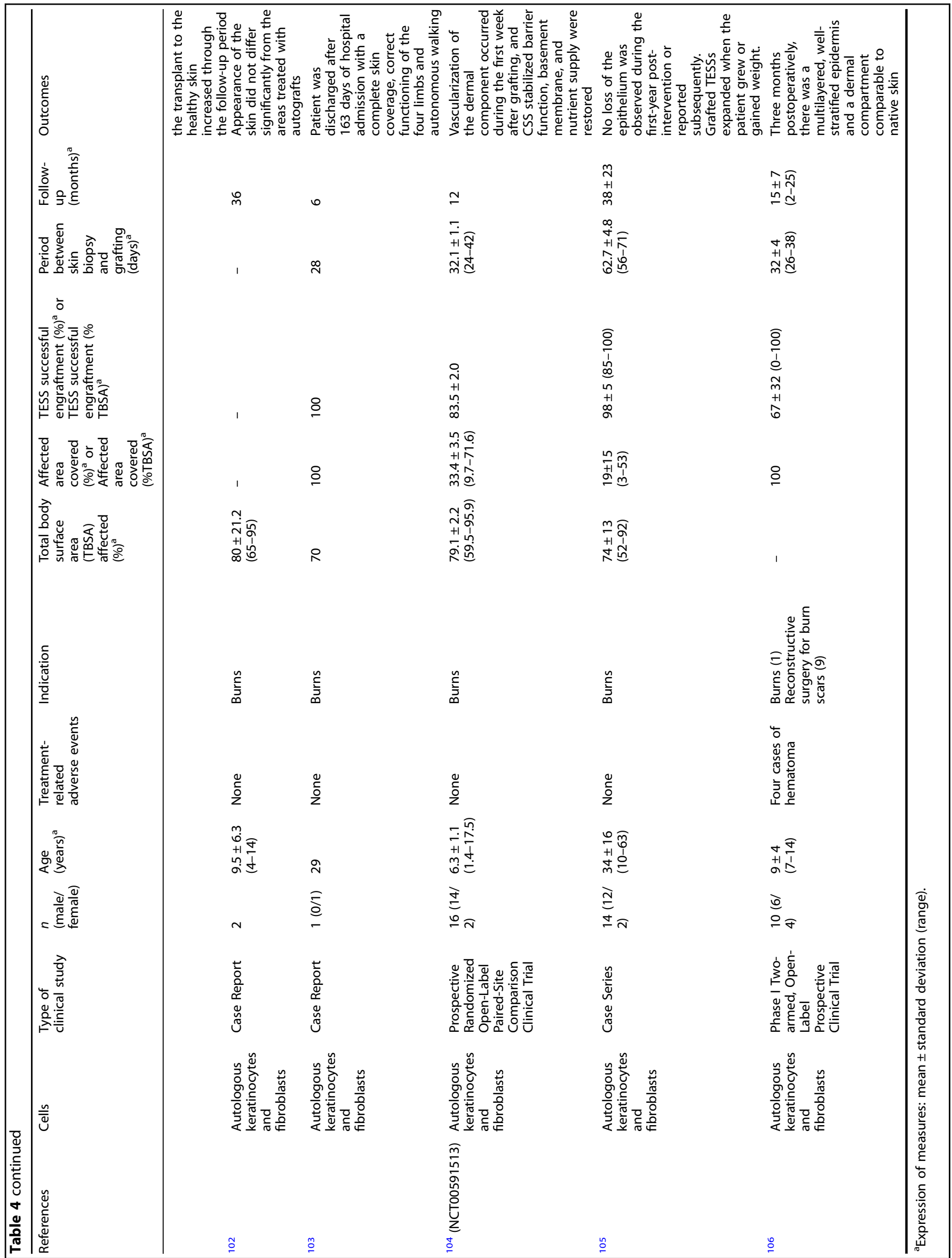




\section{Human Langerhans and Merkel cells for preclinical TESSs}

Langerhans cells are a specialized population of dendritic cells that are found in the stratum spinosum of epidermis of the skin. They help to drive protective immune responses following infection of the $\operatorname{skin}^{114}$. Merkel cells constitute a unique population of postmitotic cells scattered along the dermoepidermal junction. These cells have synaptic contacts with somatosensory afferents and play a crucial role in sensory discernment ${ }^{115}$. The number of studies evaluating the use of these cell types for TESSs is limited.

Isolation of Langerhans cells (LCS) is a complicated process. For this reason, only two studies have reported the use of in vitroderived LCs from monocytes ${ }^{116}$ or an acute myeloid leukemia cell line, MUTZ- $3^{117}$, for fabrication of collagen-based TESSs. In both cases, substitutes were composed of human fibroblasts, keratinocytes, and derived LCs. After 11-14 days of in vitro culture, histological evaluation featured a fully stratified epidermis with all the characteristic epidermal strata. Langerin-positive cells were detected suprabasally within the epidermis indicating that keratinocytes provide environmental conditions for long-time maintenance of derived LCs.

Other related studies have reported the presence of Langerhans cells (CD1a+ and human leukocyte antigen-HLA+) in vitro or in vivo in TESSs when epithelial cells were incorporated ${ }^{118-121}$. This indicates that isolation of keratinocytes, are likely to contain a small proportion of cells that are LCs.

In the case of Merkel cells, only Hahn et al. ${ }^{122}$ have reported on immunodeficient mice, the presence of host nerve cells and Merkel cells (keratin $20+-\mathrm{K} 20+-$ and $\mathrm{HLA}+$ ) from grafted human keratinocytes and fibroblasts in a collagenglycosaminoglycan scaffold, suggesting that fine touch sensation may be restored after TESS's engraftment.

\section{HUMAN STEM CELLS (hSSCS) IN TESSs}

Human stem cells such as hSSCs, induced pluripotent stem cells (hiPSCs) and hMSCs have been investigated for therapeutic use to enhance wound healing $21,27,123$. This has led to the fabrication of more complex models of TESSs (Fig. 3), which could stimulate more rapid and complete healing; furthermore, drive expression of additional phenotypes to correct anatomic deficiencies through activation of biological signaling pathways ${ }^{27}$.

\section{Human skin stem cells (hSSCs) for preclinical TESSs}

The skin is an attractive source of stem cells because of their abundant supply, easy accessibility, ease of harvesting, and possibly providing immune-privileged cells ${ }^{124}$. hSSCs, characterized by their quiescence state (CD71- EGF-R ${ }^{\text {low }}$ ) and their strong adhesion capacity (high expression of integrin markers) ${ }^{125}$, have been isolated from different parts of skin such as dermal papilla or hair follicles, among others ${ }^{12,124,126,127}$. In particular, dermal papilla stem cells (DPSCs), also called dermal hMSCs, have similar characteristics and differentiation capacity as hMSCs from other tissues $^{127}$, and for this reason they are the hSSCs most studied for skin regeneration and wound repair.

Jeremias et al. ${ }^{128}$ integrated skin-derived hMSCs with different dermal substitutes (Integra and Pelnac ${ }^{\mathrm{TM}}$ ) and showed that both were able to support the maintenance and growth of skin-derived hMSCs. Salerno et al. ${ }^{129}$ also evaluated the use of these cells in dermo-epidermal skin substitutes constituted of dermal membranes of chitosan, polycaprolactone and a polymeric blend and, after 14 days of in vitro culture, were capable of observing fibronectin deposits, as a result of dermal differentiation.

Relationship between human DPSCs and hair follicle stem cells (FSCs) has been studied in TESSs by constructing a composite based on a porcine acellular matrix with DPSCs and FSCs in the dermal and epidermal layers, respectively. This composite, when grafted in nude mice with full-thickness skin wounds, resulted in successful integration (less contraction), vascularization (higher expression of VEGF), and the presence of DPSCs-induced formation of hair buds (hair-specific keratin $6-\mathrm{K} 6 \mathrm{hf}+)^{130}$.

Another study developed by Higgins et al. ${ }^{131}$ compared human dermal fibroblasts, DPSCs and FSCs within a collagen scaffold. In vitro and in vivo experiments on nude mice revealed that both, DPSCs and FSCs, can replace interfollicular fibroblasts in skin constructs. Regarding basement membrane formation, DPSCs were found to be superior to fibroblasts with an increased type IV collagen and VEGF expression, coinciding with a formation of a more robust and uniform basal lamina.

Apart from FSCs being used in combination with DPSCs, where a correct stratification, differentiation and well-ordered epithelia was observed ${ }^{130}$, Mohd Hilmi et al. ${ }^{132}$ reported that a chitosanTESS composed of fibroblasts and FSCs serving as the epidermal component, was capable of restoring rat skin after radiation exposure by an increasing collagen bundle deposition.

The role of dermal hMSCs and other types of hSSCs in wound healing has been compared with human adipose tissue-derived MSCs (hAT-MSCs) ${ }^{133,134}$. Michalak-Micka et al. ${ }^{133}$ fabricated different collagen-based TESSs constituted of human keratinocytes as epidermal layer and stromal cells from different sources. These substitutes were evaluated in vivo in an immuneincompetent rat model and results revealed that all types of transplants exhibited a multilayered stratified epidermis with a thick stratum corneum. However, an enhanced expression of tropoelastin (a soluble precursor of elastic fibers) were only observed in skin grafts containing hAT-MSCs and dermal hMSCs, which correlated with in vivo results of Zomer et al. ${ }^{134}$, demonstrating their potential to accelerate wound healing.

\section{Human-induced pluripotent stem cells (hiPSCs) for preclinical TESSs}

Human-induced pluripotent stem cells (hiPSCs) are stem cells generated from individual somatic cells by exogenous expression of several transcription factors to initiate the reprogramming process $^{135}$. In skin regeneration and for TESSs, hiPSCs have been successfully obtained from fibroblasts ${ }^{136-138}$ or cord blood mononuclear cells $(C B M C s)^{139}$, and differentiated into fibroblasts and keratinocytes ${ }^{136-139}$

Itoh et al. ${ }^{136}$ described one of the first models of skin substitute using hiPSCs for the treatment of RDEB. They differentiated embryoid bodies generated from hiPSCs into fibroblasts and keratinocytes and showed that the hiPSCs-derived fibroblasts were capable of producing and secreting mature type VII collagen in addition to expressing other collagen types (I, III, and IV). Moreover, they fabricated and engrafted in mice a TESS constituted of collagen I matrix and these hiPSCs-derived fibroblasts, demonstrating their capacity to support functional and terminal differentiation of human keratinocytes by the expression of $\mathrm{K} 1$ and loricrin. In all, they were able to fabricate a complete hiPSCs-derived skin composite, histologically like normal human skin.

The same group was also able to differentiate hiPSCs into melanocytes ${ }^{137}$ and demonstrate that hiPSCs-derived keratinocytes, which expressed $\mathrm{K} 1$ and $\mathrm{K} 14$, were capable of internalizing melanosomes, essential to generate a functional epidermalmelanin unit.

To support these results, Petrova et al. ${ }^{138}$ focused on hiPSCsderived keratinocyte differentiation and developed a physiological purification method, which resulted in higher yield isolation of a cell population similar to normal human keratinocytes expressing $\mathrm{K} 14$ and p63. After their characterization, these derived cells were used in an in vitro TESS model demonstrating their capacity to form the same structure as the human epidermis and also, 


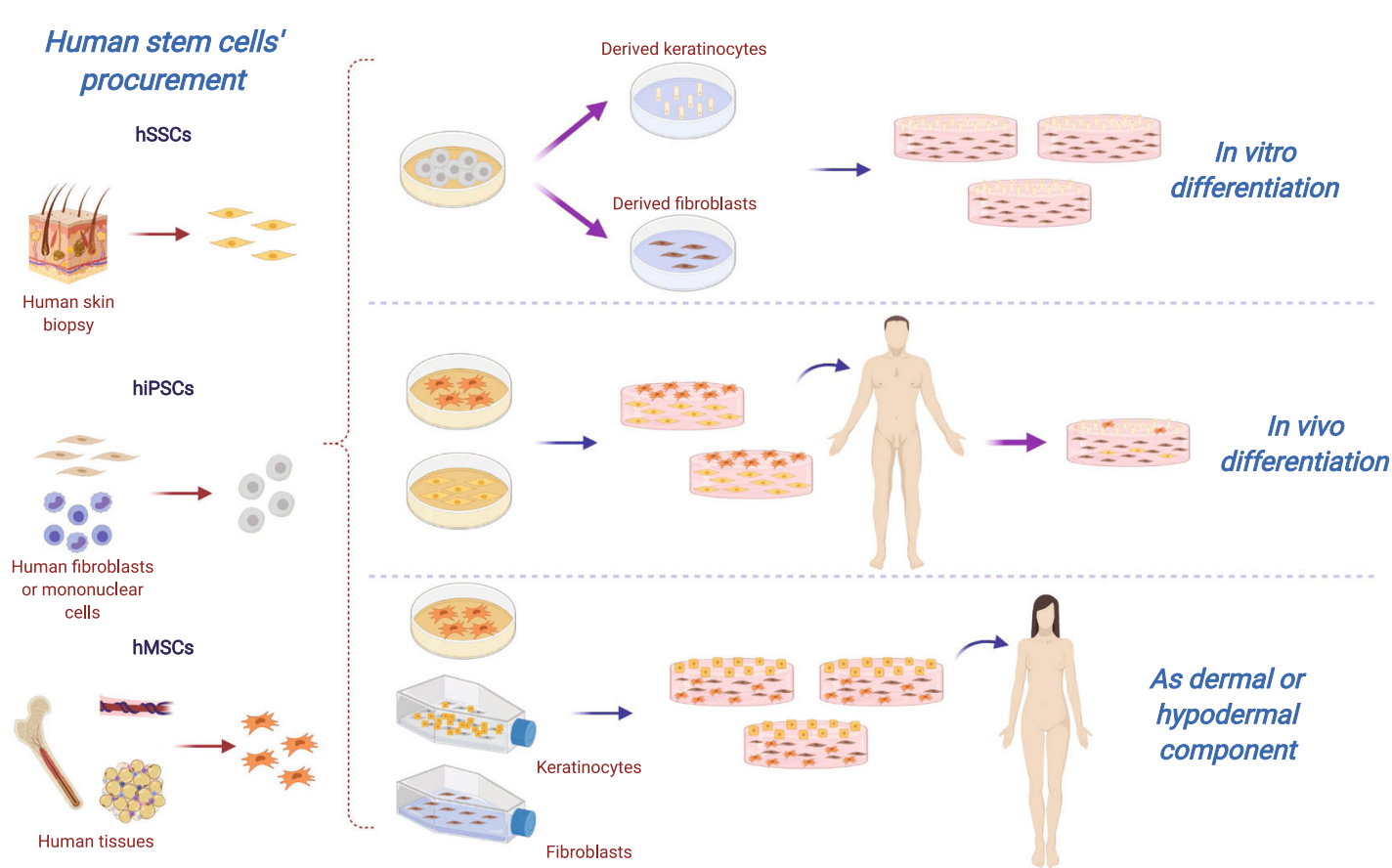

Fig. 3 Human stem cells' (hSCs) strategies for tissue-engineered skin substitutes (TESSs). Different sources of hSCs could be (i) differentiated in vitro to the main cutaneous lineages and then, uses to fabricate artificial skin; (ii) embedded directly into dermal scaffolds and engrafted to achieve an in vivo differentiation; or (iii) combined with human keratinocytes and fibroblasts to benefit from their own angiogenic and immunomodulatory properties. hSSCs human skin stem cell, hiPSCs human-induced pluripotent stem cells, hMSCs human mesenchymal stem cells. Created with BioRender.com.

develop endoplasmic reticulum $\mathrm{Ca}^{2+}$ store, essential for normal keratinocyte signaling and differentiation.

To improve the survival of skin grafts and avoid immune rejection, CBMCs have emerged as a potential cell source for regenerative medicine and hiPSCs. One advantage of CBMCs as a source is the mandatory HLA typing during the CBMC banking process, making available valuable HLA-matched research materials that can be obtained easily ${ }^{139}$. Kim et al. ${ }^{139}$ successfully differentiated hiPSCs from CBMCs into fibroblasts and keratinocytes. These cells were used to produce 3D skin organoids, and after being implanted onto surgical excisions in mice, they resembled skin structure with a similar expression of CD73 and CD105 as primary fibroblasts; and involucrin and loricrin (epidermal differentiation markers) were upregulated.

In addition to fibroblasts and keratinocytes, hiPSCs have been differentiated into other cell types such as melanocytes ${ }^{137}$, sensory neurons and Schwann cells ${ }^{140}$. They have been generated in vitro and successfully incorporated into TESSs with the purpose of fabricating more complex, functional and complete skin substitutes, although exhaustive in vivo analysis is still required.

\section{Human mesenchymal stem cells (hMSCs) for preclinical TESSs}

Mesenchymal stem cells are non-hematopoietic multipotent adult progenitor cells that are found in various tissues, including bone marrow, adipose tissue, and umbilical cord. They can be easily harvested and expanded from the different tissues of adult donors, avoiding any potential ethical issues associated with using embryonic stem cells or with genetic manipulations when using hiPSCs. Moreover, their hypo-immunogenic property allows its immediate use as prepared allogeneic cells without significant host reaction ${ }^{141-144}$, although recent studies have indicated that the immune compatibility between donor and recipient is also important because hMSCs are immune evasive rather than immune privileged ${ }^{145-147}$. Their anti-inflammatory capacity ${ }^{148}$ can also be useful in dampening the inflammatory milieu of chronic non-healing wounds and aid in the healing process.
Another beneficial feature of hMSCs is their plasticity to differentiate into both mesenchymal and non-mesenchymal lineages such as ectodermal keratinocyte-like cells (KLCs) ${ }^{149}$, endothelial cells, and different skin appendages, which is being investigated for skin tissue engineering and wound healing therapies $^{149,150}$.

Moreover, the addition of hMSCs to current skin substitute models can potentially promote angiogenesis by the recipient's endogenous cells via paracrine signaling with VEGF ${ }^{151}$.

Human bone marrow-derived MSCs (hBM-MSCs). hBM-MSCs have been the most studied and the major source of hMSCs. In the skin, hBM-MSCs' regenerative potential and their ability to differentiate into non-mesenchymal lineages including endothelial cells, keratinocyte-like cells, and skin appendages ${ }^{152}$, have been demonstrated to be useful for wound healing.

He et al. ${ }^{153}$ studied the use of hBM-MSCs and their capacity to differentiate in vitro and in vivo into epidermal and dermal cells. Better differentiation was observed in the case of dermal cells, in vitro. They fabricated TESSs composed of hBM-MSCs in a collagen membrane and implanted them into surgical skin wounds generated on the back of mice. After 21 days, wounds were completely healed and a differentiated epidermis and dermis were observed, demonstrating that hBM-MSCs could differentiate in the inducing microenvironment in vivo.

Ojeh et al. ${ }^{154}$ used hBM-MSCs as dermal component in a CSS model composed of de-epidermalized dermis with human keratinocytes and compared it with a traditional CSS composed of human fibroblasts and keratinocytes. In vitro results showed that a hBM-MSC model could generate a hyperproliferative epidermis that was well-differentiated.

hBM-MSCs have also been analyzed as the epidermal layer in fibrin-TESSs with a dermal layer composed of human fibroblasts ${ }^{152}$. This study compared MSCs from different human sources: bone marrow, umbilical cord Wharton's jelly (hWJ-MSCs) and adipose tissue (hAT-MSCs). In all cases, an epithelial-like layer 
was formed after the first week of culture, although after four weeks, more stratified epidermis was observed in the case of hBMMSCs and hWJ-MSCs. Moreover, after in vivo grafting in nude mice with surgical wounds, mesenchymal cell populations, mainly for hAT-MSCs substitutes, induced the generation of up to ten epithelial-like layers after 15 and 30 days, expressing keratin 5 , proteoglycans and collagen fibers, but without expression of HLA markers.

Human umbilical cord Wharton's jelly-derived MSCs (hWJ-MSCs). Perinatal stem cells such as hWJ-MSCs have been shown to have excellent proliferation and differentiation capabilities to be applied in regenerative medicine ${ }^{155}$.

Garzón et al. ${ }^{156}$ studied these cells in vitro and in vivo by using a bioactive 3D heterotypical model comprised of primary cell cultures of hWJ-MSCs and fibroblasts from oral mucosa or skin in a fibrin-agarose-based matrix as stroma substitute. Their results showed that hWJ-MSCs were unable to fully differentiate into epithelial cells in vitro. However, after in vivo grafting onto immunodeficient, athymic mice, they showed expression of epithelial differentiation and functional markers, and stratification into typical epithelial layers.

Ertl et al. ${ }^{157}$ compared hWJ-MSCs with two different human term placenta-derived mesenchymal stem cells (hP-MSCs) in an in vivo full-thickness wound model in mice. All TESSs fabricated with Matriderm ${ }^{\oplus}$ MSCs induced a faster healing and a higher number of blood vessels in the wound when compared to controls $(49 \pm 6 \%$ of wound reduction for TESSs vs. $22 \pm 7 \%$ of wound reduction for controls). In another study, Shi et al. ${ }^{158}$ employed hWJ-MSCs with skin microparticles in a murine excisional wound repair model to show multi-direction differentiation into newly formed skin and its appendages such as sebaceous glands, hair follicles and sweat glands.

Interestingly, some authors have explored the use of these silk fibroin-based TESSs combined with an injection of hWJ-MSCs at the edge of the wounds in mice. Results of this treatment indicated that collagen dermis organization was more similar to that typically observed in the normal skin of mice and diminished both innate and adaptative immune infiltrates ${ }^{159}$.

Human adipose tissue-derived MSCs (hAT-MSCS). hAT-MSCs are an attractive source for hMSCs-based construction of TESSs for their ease of harvesting and expansion in culture and versatile differentiation potential into non-mesenchymal lineages such as ectodermal $\mathrm{KLCs}^{149,160,161}$. Moreover, compared to MSCs from other sources such as the bone marrow, the procurement of hATMSCs is associated with lower morbidity and higher yield of cells ${ }^{155}$.

hAT-MSCs for TESSs have been used, in most of the cases, as a dermal component, alone or combined with other cell types. Some in vitro studies evaluated the role of hAT-MSCs as dermal support for human keratinocytes, showing after 7 days of culture, increased collagen IV expression in the epidermal-dermal junction $^{162}$ and enhanced proliferation of human keratinocytes ${ }^{163}$. These results were corroborated with an in vivo study in a third degree burn model generated in rats where the treatment with TESSs composed of hAT-MSCs and human keratinocytes on a human amniotic membrane reported faster wound regeneration and less inflammatory cell infiltration than control groups ${ }^{164}$. These TESSs have been also analyzed in murine models of fullthickness defects, demonstrating their capacity to enhance wound healing rates ${ }^{165}$ promoting angiogenesis and reepithelization $^{166,167}$.

Furthermore, favorable outcomes have also been observed when hAT-MSCs were combined with other cells to constitute the dermal matrix. TESSs composed of hAT-MSCs co-cultured with human endothelial cells in the dermal layer and human keratinocytes in the epidermal were capable of forming capillary structures in vitro ${ }^{168}$.

Interestingly, pigmented TESSs fabricated with hAT-MSCs and human fibroblasts in the dermal layer were less dark than those manufactured with fibroblasts only, which indicated that cytokines released by hAT-MSCs maintained melanocytes in an immature state where melanin synthesis was decreased ${ }^{169}$.

Another method of delivering hAT-MSCs is using adiposederived stromal vascular fraction (SVF), which not only contains MSCs, but also endothelial cells and pericytes that are key contributors to vasculature formation. Klar et al. ${ }^{170,171}$ developed a novel pre-vascularized composite skin substitute model by seeding adipose-derived SVF into a 3D fibrin hydrogel, allowing for the formation of vascular networks in the graft prior to transplantation. In this rat full-thickness wound model, there was more efficient engraftment of the transplanted skin substitute due to rapid anastomoses of the graft capillary plexus with the recipient's vasculature, epidermal regeneration with stratification, and remodeling of the dermis with low graft contraction.

\section{Human mesenchymal stem cells (hMSCs) for clinical TESSs}

Although most of the recent preclinical studies report the use of hAT-MSCs as the main stem cell for TESSs, the number of clinical studies is still limited. Only a small amount of published research has evaluated the use of hMSCs in TESSs as a therapeutic strategy for wound healing (Table 5).

Most of the studies reviewed were case reports ${ }^{172-175}$. Some researchers reported the use of hMSCs-based TESSs combined with autograft treatment ${ }^{174,175}$ and in other cases, a comparison between biomaterials with or without hMSCs was evaluated ${ }^{173,174,176,177}$.

Regarding tissue of origin, three studies evaluated the use of autologous hBM-MSCs ${ }^{172-174}$. Vojtassák et al. ${ }^{172}$ showed enhanced wound healing in one patient with chronic diabetic and venous ulcers using a composite graft fabricated with autologous skin fibroblasts on a collagen and hyaluronan membrane in combination with autologous hBM-MSCs injected and placed on the wounds. After 29 days of treatment, an increased vascularization of dermis was observed due to the differentiation potential of hMSCs into endothelial progenitor cells, which produced VEGF and bFGF.

Yoshikawa et al. ${ }^{173}$ studied a MSCs-based treatment of 20 patients with different pathologies whose acellular dermis grafting had previously failed. They combined cultured autologous hBMMSCs with a collagen sponge, which resulted in a significant improvement of wounds in 18 of 20 patients. Some wounds were treated with collagen membrane only and subcutaneous formation was not observed, in contrast to the rest of wounds treated with hMSCs, where infiltration of inflammatory cells was notable and CD34+ cells (derived from bone marrow) formed vascular endothelia.

Xu et al. ${ }^{174}$ applied a composite graft comprised of autologous hBM-MSCs embedded in decellularized allogeneic dermal matrix overlaid with autologous split-thickness skin graft for the treatment of hypertrophic scars resulting from burn injuries. Results demonstrated a better outcome with reduced contraction as compared to areas treated with split-thickness skin-graft alone.

hAT-MSCs were used in three studies ${ }^{175-177}$ : Arkoulis et al. ${ }^{175}$ and Stessuk et al. ${ }^{177}$ combined autologous hAT-MSCs with different dermal matrices (collagen-glycosaminoglycan and plasma, respectively) to treat eight patients with burn injuries or chronic ulcers. In the first case ${ }^{175}$, authors did not recommend the use of this technique routinely because autograft treatment was required, but it could be useful to use it when dealing with highly complex burns patients with contractures affecting cosmetically or functionally challenging areas. Stessuk et al. ${ }^{177}$ reported a total reepithelialization in 5 of 9 chronic ulcers and a healing rate of $74.6 \pm 32.6 \%$ after 9 days of treatment. Interestingly, one wound 


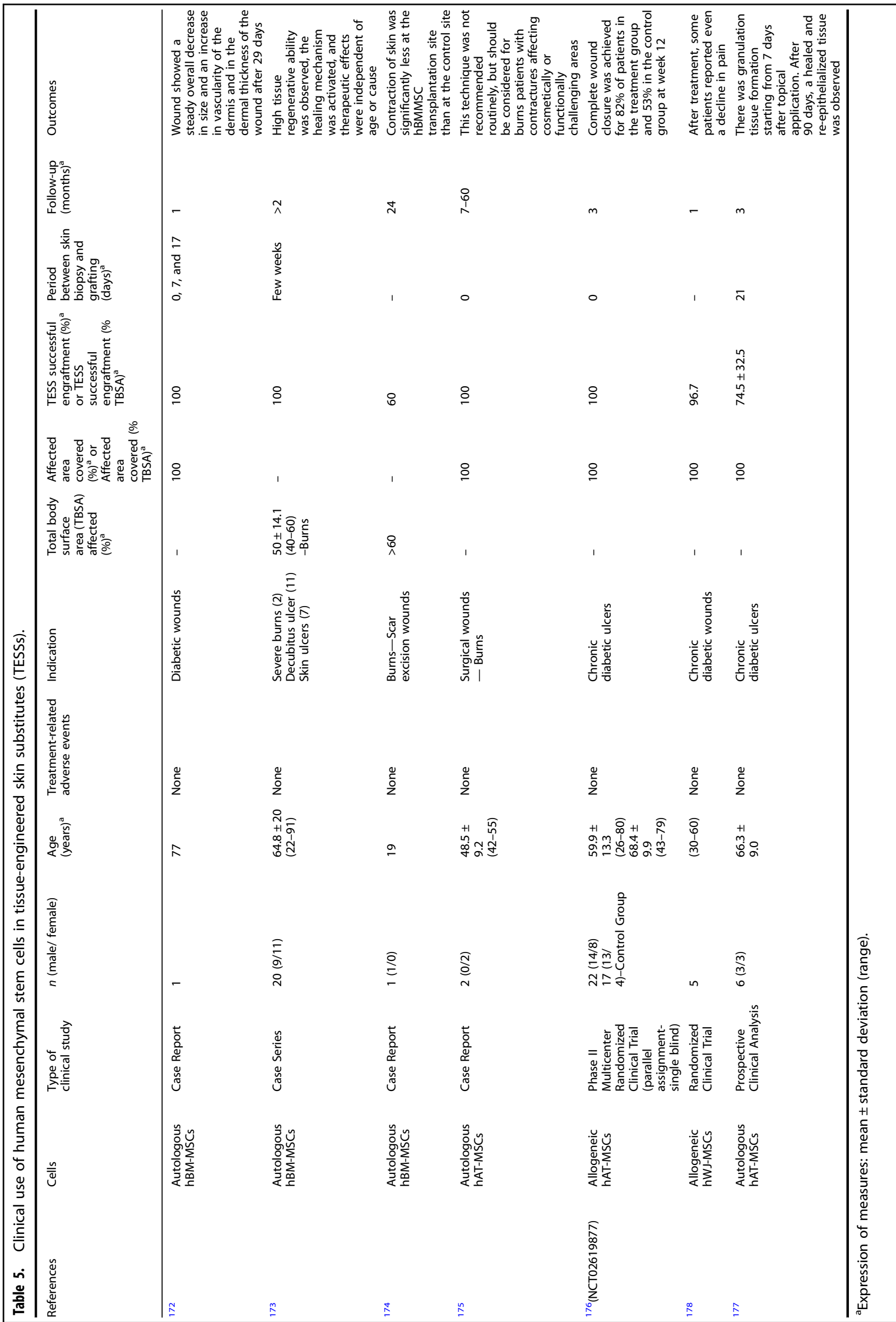


treated with plasma membrane without cells required a retreatment.

Last study that used hAT-MSCs was a phase II randomized clinical trial (NCT02619877) ${ }^{176}$ where allogeneic hMSCs embedded on a hydrogel were compared with a control group (Mepitel ${ }^{\circ}$ ), for the treatment of chronic ulcers. Results revealed that no obvious clinical rejection existed after 12 weeks (higher anti-HLA antibodies expression in $27 \%$ of the patients). Regarding to effectiveness, Kaplan-Meier median time to complete wound healing was 28.5 days for treatment group and 63.0 days for control group.

Finally, one study evaluated the use of allogeneic hWJ-MSCs in combination with amniotic membranes for the treatment of chronic ulcers ${ }^{178}$. After 9 days, wound size declined from $70.96 \mathrm{~mm}^{2}$ to $3.07 \mathrm{~mm}^{2}$ and wound healing rate was of $96.7 \%$. Moreover, patients reported decreased pain after 1 month of clinical follow up.

Overall, a total of 57 patients ( $54.4 \pm 19$ years old) with different pathologies such as burns, diabetic wounds or skin ulcers were treated with hMSCs. Burn patients had $50-60 \%$ of TBSA affected and overall successful engraftment of TESSs was $90.2 \pm 16.3 \%$ without adverse events. Interestingly, five of the seven studies investigated used autologous cells (Table 5).

\section{FUTURE APPROACH: HUMAN IMMUNE CELLS IN TESSS}

As previously described, participation of immune cells, such as neutrophils, macrophages or mast cells, in wound healing of skin is essential due their dual role as pro-inflammatory cells in first stages, and as anti-inflammatory effectors when safety is ensured ${ }^{3}$.

These cells are capable of phagocyte cell debris, synthetize or release several cytokines, which promotes angiogenesis and wound healing (VEGF), activate keratinocyte's proliferation, and re-epithelialization or induce fibroblasts transition into myofibroblasts to increase ECM and collagen deposition ${ }^{3,10}$. Moreover, recent studies have indicated that immune cells are members of stem cells niches, developing a proactive role in regulating stem cells when tissues are damaged ${ }^{10,179,180}$.

Macrophages and Foxp3+CD4+ regulatory T (Treg) cells seems to be the most important immune cell populations involved in this context $^{10,179,180}$. Macrophages are capable of sensing the metabolic environment ${ }^{181}$ and therefore, modulating stem cells function ${ }^{179}$, meanwhile, Treg cells infiltrated in wounds express the epidermal growth factor receptor (EGF-R), which is related with an improvement of wound healing ${ }^{180}$.

In wound healing of skin when regenerative phases are triggered, macrophages around the follicle die off and release factors such as WNT7b and WNT10a, which promote the activation of $\mathrm{FSCs}^{182}$. Moreover, these macrophages physically contact with epithelial stem cells, secreting pro-proliferative and epithelial remodeling factors such as IL-10 and PDGF- $\beta^{183}$, apart from TGF$\beta 1$, which induces fibroblast proliferation and their differentiation into myofibroblasts ${ }^{184}$.

In the case of Treg cells, they are predominantly localized around hair follicles in contact to FSCs. Their role in skin regeneration could be interested for lesions, which affect epidermal appendages such as hair follicles ${ }^{179,180}$. Several researches have studied the link between Treg cells and hair follicles biology, demonstrating that in alopecia areata patients the number of Foxp3+ Treg cells is reduced in comparison with healthy controls ${ }^{185}$. In addition, stimulation of Treg proliferation with IL-2 administration demonstrated successful hair regeneration in $80 \%$ of patients ${ }^{186}$, which is due to the expression of the Notch ligand Jagged-1 (Jag1), required to promote hair follicle cycling by enhancing the activation and differentiation of FSCs ${ }^{180}$.

For all of this, incorporation of human immune cells in TESSs, alone or combined with other cell populations might be interesting to increase regeneration potential and develop more complex models of artificial skin, which included epidermal appendages such as hair follicles. However, no preclinical research has been published yet in this field, which is essential to ensure their wound healing's safety and effectiveness.

\section{ALLOGENEIC CELLS: A REAL STRATEGY FOR CLINICAL TESSs?}

In addition to determining the cell composition of TESSs, for clinical purpose, the selection between allogeneic or autologous cells is a critical decision. While the use of allogeneic cells ensures quicker availability, graft survival is usually short-term $(4-8 \text { weeks })^{187}$. The use of autologous source avoids any possible host rejection and permits a more permanent TESS for fullthickness burns and chronic wounds ${ }^{95}$. The downside of autologous cells is the longer period ( $\sim 4$ weeks) required to produce a sufficiently sized graft.

Interestingly, many of the TESSs developed for clinical use (Tables 2-5) were constituted of autologous cells, mainly for CESs, CSSs or hMSC-based TESSs, with only five studies reporting the use of allogeneic cells $s^{55,90,93,176,178}$. However, in the case of CDSs, the use of allogeneic fibroblasts was preferred against autologous fibroblasts ${ }^{66,67}$, which could be explained by their use as temporary dressing to prepare the wound's bed for future therapies or the small size of wounds treated, when a shortterm biological recovery dictates the long-term outcomes ${ }^{147}$ (Table 3).

In the case of adult skin cells, the use of autologous populations for TESSs seems to be clear, to avoid rapid rejection. However, when allogeneic hMSCs are selected, there is a controversy because their immunogenicity have been proven ${ }^{188}$, but no acute adverse events have been reported when were applied as therapy in several pathologies ${ }^{146}$.

To avoid this concern, immunosuppression treatment could be effective for the use of allogeneic cells in many clinical conditions, however, this poses a risk for long-term therapies where other pathologies could be developed due to a continuous suppression of immune system and moreover, in the case of skin or TESS transplants, is either less or/not effective ${ }^{189}$.

On balance, although immune rejection of allogeneic hMSCs occurs more slowly than other cell types ${ }^{145,188}$, they are immune evasive rather than immune privileged and for this reason, it could be interesting to use haplo-identical hMSCs ${ }^{145,147}$ to increase the potential benefits of allogeneic TESSs when autologous approach is not possible.

\section{MAIN BIOMATERIALS FOR CLINICAL TESSS}

Many different biomaterials have been investigated for the development of TESSs; from xenogeneic scaffolds such as porcine acellular matrix ${ }^{130}$, natural polymers like silk fibroin ${ }^{159}$, agarose $^{32,156}$ or chitosan ${ }^{29,129,132}$ to substances that resemble in better way the native dermal components of skin: collagen ${ }^{56,60-65,86-}$ $93,95,101,104,106$, plasma/fibrin $49,51,94,96,98,102,103$, hyaluronic acid ${ }^{60-}$ 65,97 , elastin ${ }^{56}$, amniotic membrane ${ }^{68,178}$ or extracellular matrix derived from fibroblasts $39,99,100,105$.

For clinical purposes, the main biomaterials used have been collagen alone or combined with glycosaminoglycan, hyaluronic acid, plasma/fibrin, amniotic membranes and acellular dermal matrices (Table 6).

Collagen is the most abundant of animal proteins, localized in soft and hard connective tissues where its fibrils, with high tensile strength and stability via cross-linking, comprise the majority of ECM and form a highly organized, 3D scaffold that surrounds the cells. Moreover, it is a dynamic and flexible biomaterial (used as sponge, gel or membrane) with high biocompatibility and intrinsic biodegradability ideal for biomedical applications ${ }^{190}$. Glycosaminoglycan is a negatively charged polysaccharide and one of the most prevalent crosslinks of collagen, that affects their mechanical 
properties and fibril formation ${ }^{191}$. The main aspect to consider the incorporation into TESSs is the presence of negatively charged carboxyl and sulfate groups that are responsible of maintaining water in tissues ${ }^{192}$, and therefore, skin barrier.

Hyaluronic acid is also an important component of human skin ${ }^{193,194}$ and its use for TESSs is recommended. It is easy to handle, their biosafety has been corroborated by its use as injectable dermal fillers ${ }^{195}$, and its effectiveness have been demonstrated for skin restoration in terms of hydration and transepidermal water loss ${ }^{196}$, mainly when the water regulation and neoangiogenic boost are relevant issues ${ }^{197}$. Moreover, biodegraded hyaluronic acid, enhances angiogenic pathways and the migration and proliferation of cells ${ }^{198}$.

In the case of plasma/fibrin-based matrices, their use for TESSs have reported positive results due to the presence of many natural components responsible of coagulation and involved in the first stages of wound healing. It has been demonstrated that enhances cell proliferation (keratinocytes mainly), due to the presence of basement membrane proteins such as laminin, collagen or Perlecan ${ }^{199}$.

Finally, amniotic membrane and acellular dermal matrix are the less common biomaterials used for TESSs, however, they also provide structural support and secrete important proteins or factors required for wound healing. Amniotic membrane has high tensile strength due to a structure comprised of an epithelial monolayer, a thick basement membrane and avascular stroma. In addition, it releases several growth factors for angiogenesis, downregulates TGF- $\beta$ expression, promotes fibroblast differentiation and keratinocytes proliferation, and reduces levels of pain and discomfort experienced by the patients ${ }^{200}$. The use of acellular dermal matrix derived from fibroblast is an interested strategy because the use of natural ECM shares many properties with native human skin and minimizes the host response after transplantation $^{78}$, but specific formation is required to carry out it successfully.

\section{DISCUSSION}

Notwithstanding the tremendous advances in skin tissue engineering, we have yet to construct a complete TESS. Current substitutes are mainly composed of human keratinocytes and fibroblasts, but still lack some of the functional components such as nerves, adnexal structures and pigmentary cells that make up the native skin, and the esthetic and functional outcome is less than ideal.

Moreover, to develop an ideal TESS that could be applied at clinical level, the use of appropriate wound and animal preclinical models is essential. In this review, most of the in vivo studies evaluated excisional or burn wounds in mice or rats. The use of these animals together with other small mammals such as rabbit or guinea pig is due to their cost and easy to handle, however, their anatomical and physiological skin properties and wound healing process differ from the humans (for example, thin epidermis and dermis and heal primarily through wound contraction instead of re-epithelialization) ${ }^{201}$.

After analyzing their properties, many authors suggested that the use of pigs should be the preferred animal model ${ }^{201-204}$. Among similarities reported, epidermis and dermis of human and pig skin are comprised of four and two layers, respectively, without significant differences of thickness, and dermo-epidermal junction has an undulating appearance ${ }^{204}$. In terms of functionality, permeability is also similar ${ }^{204}$. However, the use of this model is expensive and more difficult due to their size and for this reason the number of studies with human TESSs is limited ${ }^{205}$ or own pig derived cells are used ${ }^{206,207}$.

Considering the types of wounds analyzed, burns or fullthickness skin injuries are the most studied models at preclinical level but also in a clinical environment. Application of commercial 
biological substitutes have been extensively reviewed ${ }^{208}$ and analyzed $^{209}$ and even the use of cell therapies have reported positive results ${ }^{210}$. However, the development of TESSs constituted of different cell types and biomaterials seems to be essential to increase skin wound healing potential.

Apart from the use of stem cells, even from burned and debrided $\operatorname{skin}^{205}$, future perspectives in the field of TESSs for wound healing are focused on the development of more similar models of artificial skin where 3D bioprinting ${ }^{211}$, designing stem cell niches ${ }^{212}$ or incorporation of immune cells ${ }^{10,179,180}$ will play an important role.

\section{CONCLUSION}

To date, the positive clinical results obtained with autologous and allogeneic TESSs based on human adult skin cells and hMSCs, regarding successful engraftment (60-90\% in most of the studies), safety (slight adverse events in some cases), re-epithelialization and wound healing rates, are promising. However, if we improve current techniques such as the selection of an appropriate animal model and biomaterial or application of 3D bioprinting and expand the toolset with innovative strategies based on ever expanding understanding of skin healing and regeneration (immune cells or stem cell niches), the fabrication of a more functional and physiological TESS, which is clinically beneficial and esthetically acceptable to our patients, is not beyond reach.

\section{DATA AVAILABILITY}

No datasets were generated or analyzed during the current study.

Received: 27 September 2020; Accepted: 25 May 2021; Published online: 17 June 2021

\section{REFERENCES}

1. Naves, L. B., Dhand, C., Almeida, L., Rajamani, L. \& Ramakrishna, S. In vitro skin models and tissue engineering protocols for skin graft applications. Essays Biochem. 60, 357-369 (2016).

2. Reinke, J. M. \& Sorg, H. Wound repair and regeneration. Eur. Surg. Res. 49, 35-43 (2012).

3. Rodrigues, M., Kosaric, N., Bonham, C. A. \& Gurtner, G. C. Wound healing: a cellular perspective. Physiol. Rev. 99, 665-706 (2019).

4. Berk, B. C., Alexander, R. W., Brock, T. A., Gimbrone Jr., M. A. \& Webb, R. C. Vasoconstriction: a new activity for platelet-derived growth factor. Science (80-.). 232, 87-90 (1986).

5. Pradhan, S., Khatlani, T., Nairn, A. C. \& Vijayan, K. V. The heterotrimeric G protein G1 interacts with the catalytic subunit of protein phosphatase 1 and modulates $\mathrm{G}$ protein-coupled receptor signaling in platelets. J. Biol. Chem. 292, 13133-13142 (2017).

6. Wagner, C. et al. Analysis of GPIlb/llla receptor number by quantification of 7E3 binding to human platelets. Blood 88, 907-914 (1996).

7. Santoro, S. A. Identification of a 160,000 dalton platelet membrane protein that mediates the initial divalent cation-dependent adhesion of platelets to collagen. Cell 46, 913-920 (1986).

8. Bielefeld, K. A., Amini-Nik, S. \& Alman, B. A. Cutaneous wound healing: recruiting developmental pathways for regeneration. Cell. Mol. Life Sci. 70, 2059-2081 (2013).

9. Van Der Vliet, A. \& Janssen-Heininger, Y. M. W. Hydrogen peroxide as a damage signal in tissue injury and inflammation: murderer, mediator, or messenger? J. Cell. Biochem. 115, 427-435 (2014).

10. Brazil, J. C., Quiros, M., Nusrat, A. \& Parkos, C. A. Innate immune cell-epithelial crosstalk during wound repair. J. Clin. Investig. 129, 2983-2993 (2019).

11. Dvorak, A. M. Mast cell-derived mediators of enhanced microvascular permeability, vascular permeability factor/vascular endothelial growth factor, histamine, and serotonin, cause leakage of macromolecules through a new endothelial cell permeability organelle, the vesiculo-vacuolar organelle. Chem. Immunol. Allergy 85, 185-204 (2005).

12. Stone li, R. et al. Advancements in regenerative strategies through the continuum of burn care. Front. Pharmacol. 9, 672 (2018).
13. Liu, Z.-J. et al. Regulation of Notch 1 and DIl4 by vascular endothelial growth factor in arterial endothelial cells: implications for modulating arteriogenesis and angiogenesis. Mol. Cell. Biol. 23, 14-25 (2003).

14. Hobbs, R. M., Silva-Vargas, V., Groves, R. \& Watt, F. M. Expression of activated MEK1 in differentiating epidermal cells is sufficient to generate hyperproliferative and inflammatory skin lesions. J. Invest. Dermatol. 123, 503-515 (2004).

15. Pastar, I. et al. Epithelialization in wound healing: a comprehensive review. Adv. Wound Care 3, 445-464 (2014).

16. Watt, F. M., Celso, C., Lo \& Silva-Vargas, V. Epidermal stem cells: an update. Curr. Opin. Genet. Dev. 16, 518-524 (2006).

17. Fuchs, E. Skin stem cells: rising to the surface. J. Cell Biol. 180, 273-284 (2008).

18. Ito, M. et al. Stem cells in the hair follicle bulge contribute to wound repair but not to homeostasis of the epidermis. Nat. Med. 11, 1351-1354 (2005).

19. Gurtner, G. C., Werner, S., Barrandon, Y. \& Longaker, M. T. Wound repair and regeneration. Nature 453, 314-321 (2008).

20. Vig, K. et al. Advances in skin regeneration using tissue engineering. Int. J. Mol. Sci. 18, 789 (2017).

21. Sierra-Sánchez, Á., Montero-Vilchez, T., Quiñones-Vico, M. I., Sanchez-Diaz, M. \& Arias-Santiago, S. Current advanced therapies based on human mesenchymal stem cells for skin diseases. Front. Cell Dev. Biol. 9, 643125 (2021).

22. Sharma, P., Kumar, P., Sharma, R., Bhatt, V. D. \& Dhot, P. S. Tissue engineering; current status \& futuristic scope. J. Med. life 12, 225-229 (2019).

23. Olson, J. L., Atala, A. \& Yoo, J. J. Tissue engineering: current strategies and future directions. Chonnam Med. J. 47, 1 (2011).

24. Ikada, Y. Challenges in tissue engineering. J. R. Soc. Interface 3, 589-601 (2006).

25. Sheridan, R. L. \& Tompkins, R. G. Skin substitutes in burns. Burns 25, 97-103 (1999).

26. Davison-Kotler, E., Sharma, V., Kang, N. V. \& García-Gareta, E. A universal classification system of skin substitutes inspired by factorial design. Tissue Eng. Part B Rev. 24, 279-288 (2018).

27. Boyce, S. T. \& Lalley, A. L. Tissue engineering of skin and regenerative medicine for wound care. Burn. Trauma 6, 4 (2018).

28. Dong, C., Lv, Y., Dong, C. \& Lv, Y. Application of collagen scaffold in tissue engineering: recent advances and new perspectives. Polymers (Basel). 8, 42 (2016).

29. Mohd Hilmi, A. B., Halim, A. S., Jaafar, H., Asiah, A. B. \& Hassan, A. Chitosan dermal substitute and Chitosan skin substitute contribute to accelerated fullthickness wound healing in irradiated rats. Biomed. Res. Int. 2013, 795458 (2013).

30. Rodríguez-Cabello, J. C., González de Torre, I., Ibañez-Fonseca, A. \& Alonso, M. Bioactive scaffolds based on elastin-like materials for wound healing. Adv. Drug Deliv. Rev. 129, 118-133 (2018).

31. Mineo, A., Suzuki, R. \& Kuroyanagi, Y. Development of an artificial dermis composed of hyaluronic acid and collagen. J. Biomater. Sci. Polym. Ed. 24, 726-740 (2013).

32. Sierra-Sánchez, Á. et al. Hyaluronic acid biomaterial for human tissueengineered skin substitutes: Preclinical comparative in vivo study of wound healing. J. Eur. Acad. Dermatol. Venereol. 34, 2414-2427 (2020).

33. Chaudhari, A. A. et al. Future prospects for scaffolding methods and biomaterials in skin tissue engineering: a review. Int. J. Mol. Sci. 17, 1974 (2016).

34. Rheinwald, J. G. \& Green, H. Serial cultivation of strains of human epidemal keratinocytes: the formation of keratinizing colonies from single cells. Cell 6, 344 (1975).

35. Chocarro-Wrona, C., López-Ruiz, E., Perán, M., Gálvez-Martín, P. \& Marchal, J. A. Therapeutic strategies for skin regeneration based on biomedical substitutes. $J$. Eur. Acad. Dermatol. Venereol. 33, 484-496 (2019).

36. Dill, V. \& Mörgelin, M. Biological dermal templates with native collagen scaffolds provide guiding ridges for invading cells and may promote structured dermal wound healing. Int. Wound J. 13314 https://doi.org/10.1111/iwj.13314 (2020).

37. Kalyanaraman, B. \& Boyce, S. T. Wound healing on athymic mice with engineered skin substitutes fabricated with keratinocytes harvested from an automated bioreactor. J. Surg. Res. 152, 296-302 (2009).

38. Ghetti, M. et al. Subpopulations of dermal skin fibroblasts secrete distinct extracellular matrix: implications for using skin substitutes in the clinic. Br. J. Dermatol. 179, 381-393 (2018).

39. Rennekampff, H. O., Kiessig, V., Griffey, S., Greenleaf, G. \& Hansbrough, J. F. Acellular human dermis promotes cultured keratinocyte engraftment. J. Burn Care Rehabil. 18, 535-544 (1997).

40. Horch, R. E. et al. Keratinocyte monolayers on hyaluronic acid membranes as 'upside-down' grafts reconstitute full-thickness wounds. Med. Sci. Monit. 25, 6702-6710 (2019).

41. O'Connor, N. E., Mulliken, J. B., Banks-Schlegel, S., Kehinde, O. \& Green, H. Grafting of burns with cultured epithelium prepared from autologous epidermal cells. Lancet 317, 75-78 (1981).

42. Teepe, R. G. C. et al. The use of cultured autologous epidermis in the treatment of extensive burn wounds. J. Trauma 30, 269-275 (1990). 
43. Blight, A., Mountford, E. M., Cheshire, I. M., Clancy, J. M. P. \& Levick, P. L. Treatment of full skin thickness burn injury using cultured epithelial grafts. Burns 17, 495-498 (1991).

44. Donati, L., Magliacani, G., Bormioli, M., Signorini, M. \& Baruffaldi Preis, F. W. Clinical experiences with keratinocyte grafts. Burns 18, S19-S26 (1992).

45. Rue, L. W., Cioffi, W. G., McManus, W. F. \& Pruitt, B. A. Wound closure and outcome in extensively burned patients treated with cultured autologous keratinocytes. J. Trauma 34, 662-668 (1993).

46. Hickerson, W. L., Compton, C., Fletchall, S. \& Smith, L. R. Cultured epidermal autografts and allodermis combination for permanent burn wound coverage. Burns 20, S52-S55 (1994).

47. Williamson, J. S., Snelling, C. F., Clugston, P., Macdonald, I. B. \& Germann, E. Cultured epithelial autograft: five years of clinical experience with twenty-eight patients. J. Trauma 39, 309-319 (1995).

48. Paddle-Ledinek, J. E., Cruickshank, D. G. \& Masterton, J. P. Skin replacement by cultured keratinocyte grafts: an Australian experience. Burns 23, 204-211 (1997).

49. Pellegrini, G. et al. The control of epidermal stem cells (holoclones) in the treatment of massive full-thickness burns with autologous keratinocytes cultured on fibrin. Transplantation 68, 868-879 (1999).

50. Barret, J. P., Wolf, S. E., Desai, M. H. \& Herndon, D. N. Cost-efficacy of cultured epidermal autografts in massive pediatric burns. Ann. Surg. 231, 869-876 (2000).

51. Ronfard, V., Rives, J. M., Neveux, Y., Carsin, H. \& Barrandon, Y. Long-term regeneration of human epidermis on third degree burns transplanted with autologous cultured epithelium grown on a fibrin matrix. Transplantation 70, 1588-1598 (2000).

52. Yamaguchi, Y. et al. Epithelial-mesenchymal interactions in wounds: treatment of palmoplantar wounds by nonpalmoplantar pure epidermal sheet grafts. Arch. Dermatol. 137, 621-628 (2001).

53. Sheridan, R. L. et al. Initial experience with a composite autologous skin substitute. Burns 27, 421-424 (2001).

54. Elliott, M. \& Vandervord, J. Initial experience with cultured epithelial autografts in massively burnt patients. ANZ J. Surg. 72, 893-895 (2002).

55. Pajardi, G. et al. Skin substitutes based on allogenic fibroblasts or keratinocytes for chronic wounds not responding to conventional therapy: a retrospective observational study. Int. Wound J. 13, 44-52 (2016).

56. Gardien, K. L. M. et al. Outcome of burns treated with autologous cultured proliferating epidermal cells: a prospective randomized multicenter intrapatient comparative trial. Cell Transplant. 25, 437-448 (2016).

57. Łabuś, W. et al. Atomic force microscopy in the production of a biovital skin graft based on human acellular dermal matrix produced in-house and in vitro cultured human fibroblasts. J. Biomed. Mater. Res. Part B Appl. Biomater. 106, 726-733 (2018)

58. Jang, H. J., Kim, Y. M., Yoo, B. Y. \& Seo, Y. K. Wound-healing effects of human dermal components with gelatin dressing. J. Biomater. Appl. 32, 716-724 (2018).

59. Mazio, $C$. et al. Pre-vascularized dermis model for fast and functional anastomosis with host vasculature. Biomaterials 192, 159-170 (2019).

60. Kashiwa, N. et al. Treatment of full-thickness skin defect with concomitant grafting of 6-fold extended mesh auto-skin and allogeneic cultured dermal substitute. Artif. Organs 28, 444-450 (2004).

61. Hasegawa, T. et al. An allogeneic cultured dermal substitute suitable for treating intractable skin ulcers and large skin defects prior to autologous skin grafting: three case reports. J. Dermatol. 32, 715-720 (2005).

62. Yonezawa, M. et al. Clinical study with allogeneic cultured dermal substitutes for chronic leg ulcers. Int. J. Dermatol. 46, 36-42 (2007).

63. Yamada, N., Uchinuma, E., Matsumoto, Y. \& Kuroyanagi, Y. Comparative evaluation of re-epithelialization promoted by fresh or cryopreserved cultured dermal substitute. J. Artif. Organs 11, 221-224 (2008).

64. Yamada, N., Uchinuma, E. \& Kuroyanagi, Y. Clinical trial of allogeneic cultured dermal substitutes for intractable skin ulcers. J. Artif. Organs 15, 193-199 (2012).

65. Taniguchi, T., Amoh, Y., Tanabe, K., Katsuoka, K. \& Kuroyanagi, Y. Treatment of intractable skin ulcers caused by vascular insufficiency with allogeneic cultured dermal substitute: a report of eight cases. J. Artif. Organs 15, 77-82 (2012).

66. You, H. J., Han, S. K. \& Rhie, J. W. Randomised controlled clinical trial for autologous fibroblast-hyaluronic acid complex in treating diabetic foot ulcers. $J$. Wound Care 23, 521-530 (2014).

67. Morimoto, N. et al. An exploratory clinical study on the safety and efficacy of an autologous fibroblast-seeded artificial skin cultured with animal product-free medium in patients with diabetic foot ulcers. Int. Wound J. 11, 183-189 (2014).

68. Momeni, M. et al. A randomized, double-blind, phase I clinical trial of fetal cellbased skin substitutes on healing of donor sites in burn patients. Burns 45, 914-922 (2019).

69. Sun, B. K., Siprashvili, Z. \& Khavari, P. A. Advances in skin grafting and treatment of cutaneous wounds. Science 346, 941-945 (2014).
70. Bell, E., Ehrlich, H. P., Buttle, D. J. \& Nakatsuji, T. Living tissue formed in vitro and accepted as skin-equivalent tissue of full thickness. Science 211, 1052-1054 (1981).

71. Pontiggia, L. et al. Markers to evaluate the quality and self-renewing potential of engineered human skin substitutes in vitro and after transplantation. J. Invest. Dermatol. 129, 480-490 (2009).

72. Hartmann-Fritsch, F. et al. Collagen hydrogels strengthened by biodegradable meshes are a basis for dermo-epidermal skin grafts intended to reconstitute human skin in a one-step surgical intervention. J. Tissue Eng. Regen. Med. 10, 81-91 (2016).

73. Supp, D. M. et al. Collagen VII expression is required in both keratinocytes and fibroblasts for anchoring fibril formation in bilayer engineered skin substitutes. Cell Transplant. 28, 1242-1256 (2019).

74. Bacakova, M. et al. A two-layer skin construct consisting of a collagen hydrogel reinforced by a fibrin-coated polylactide nanofibrous membrane. Int. J. Nanomed. 14, 5033-5050 (2019).

75. L'Heureux, N. et al. A human tissue-engineered vascular media: a new model for pharmacological studies of contractile responses. FASEB J. 15, 515-524 (2001).

76. Michel, $M$. et al. Characterization of a new tissue-engineered human skin equivalent with hair. In Vitro Cell. Dev. Biol. Anim. 35, 318-326 (1999).

77. Beaudoin Cloutier, C. et al. Production of a bilayered self-assembled skin substitute using a tissue-engineered acellular dermal matrix. Tissue Eng. -Part C. Methods 21, 1297-1305 (2015).

78. Larouche, D. et al. Improved methods to produce tissue-engineered skin substitutes suitable for the permanent closure of full-thickness skin injuries. Biores. Open Access 5, 320-329 (2016).

79. Beaudoin Cloutier, $C$. et al. In vivo evaluation and imaging of a bilayered selfassembled skin substitute using a decellularized dermal matrix grafted on mice. Tissue Eng. 23, 313-322 (2017).

80. Cantin-Warren, L. et al. Specialized living wound dressing based on the selfassembly approach of tissue engineering. J. Funct. Biomater. 9, 53 (2018).

81. bin Mh Busra, M. F., Chowdhury, S. R., bin Ismail, F., bin Saim, A. \& Idrus, R. H. Tissue-engineered skin substitute enhances wound healing after radiation therapy. Adv. Ski. Wound Care 29, 120-129 (2016).

82. Klar, A. S., Biedermann, T., Simmen-Meuli, C., Reichmann, E. \& Meuli, M. Comparison of in vivo immune responses following transplantation of vascularized and non-vascularized human dermo-epidermal skin substitutes. Pediatr. Surg. Int. 33, 377-382 (2017)

83. Asano, Y., Shimoda, H., Okano, D., Matsusaki, M. \& Akashi, M. Transplantation of three-dimensional artificial human vascular tissues fabricated using an extracellular matrix nanofilm-based cell-accumulation technique. J. Tissue Eng. Regen. Med. 11, 1303-1307 (2017).

84. Dai, N. T. et al. Development of a novel pre-vascularized three-dimensional skin substitute using blood plasma gel. Cell Transplant. 27, 1535-1547 (2018).

85. Miyazaki, $\mathrm{H}$. et al. A novel strategy to engineer pre-vascularized 3-dimensional skin substitutes to achieve efficient, functional engraftment. Sci. Rep. 9, 7797 (2019).

86. Hansbrough, J. F., Boyce, S. T., Cooper, M. L. \& Foreman, T. J. Burn wound closure with cultured autologous keratinocytes and fibroblasts attached to a collagenglycosaminoglycan substrate. JAMA J. Am. Med. Assoc. 262, 2125-2130 (1989).

87. Kuroyanagi, Y. et al. A cultured skin substitute composed of fibroblasts and keratinocytes with a collagen matrix: preliminary results of clinical trials. Ann. Plast. Surg. 31, 340-351 (1993).

88. Boyce, S. T. et al. Comparative assessment of cultured skin substitutes and native skin autograft for treatment of full-thickness burns. Ann. Surg. 222, 743-752 (1995).

89. Dana Harriger, M., Warden, G. D., Greenhalgh, D. G., Kagan, R. J. \& Boyce, S. T. Pigmentation and microanatomy of skin regenerated from composite grafts of cultured cells and biopolymers applied to full-thickness burn wounds. Transplantation 59, 702-707 (1995).

90. Muhart, M., McFalls, S., Kirsner, R., Kerdel, F. \& Eaglstein, W. H. Bioengineered skin [2]. Lancet 350, 1142 (1997).

91. Boyce, S. T., Kagan, R. J., Meyer, N. A., Yakuboff, K. P. \& Warden, G. D. Cultured skin substitutes combined with integra artificial skin to replace native skin autograft and allograft for the closure of excised full- thickness burns. J. Burn Care Rehabilit. 20, 453-461 (1999).

92. Boyce, S. T. et al. Cultured skin substitutes reduce donor skin harvesting for closure of excised, full-thickness burns. Ann. Surg. 235, 269-279 (2002).

93. Nanchahal, J., Dover, R. \& Otto, W. R. Allogeneic skin substitutes applied to burns patients. Burns 28, 254-257 (2002).

94. Llames, S. G. et al. Human plasma as a dermal scaffold for the generation of a completely autologous bioengineered skin. Transplantation 77, 350-355 (2004).

95. Boyce, S. T. et al. Cultured skin substitutes reduce requirements for harvesting of skin autograft for closure of excised, full-thickness burns. J. Trauma 60, 821-829 (2006). 
96. Llames, S. et al. Clinical results of an autologous engineered skin. Cell Tissue Bank. 7, 47-53 (2006).

97. Scuderi, N., Anniboletti, T., Carlesimo, B. \& Onesti, M. G. Clinical application of autologous three-cellular cultured skin substitutes based on esterified hyaluronic acid scaffold: our experience. In Vivo (Brooklyn). 23, 991-1003 (2009).

98. Gómez, C. et al. Use of an autologous bioengineered composite skin in extensive burns: Clinical and functional outcomes. A multicentric study. Burns 37, 580-589 (2011).

99. Boa, O. et al. Prospective study on the treatment of lower-extremity chronic venous and mixed ulcers using tissue-engineered skin substitute made by the self-assembly approach. Adv. Ski. Wound Care 26, 400-409 (2013).

100. Takami, Y., Yamaguchi, R., Ono, S. \& Hyakusoku, H. Clinical application and histological properties of autologous tissue-engineered skin equivalents using an acellular dermal matrix. J. Nippon Med. Sch. 81, 356-366 (2014).

101. Golinski, P. et al. Development and characterization of an engraftable tissuecultured skin autograft: alternative treatment for severe electrical injuries? Cells Tissues Organs 200, 227-239 (2015).

102. Kljenak, A. et al. Fibrin gel as a scaffold for skin substitute-production and clinical experience. Acta Clin. Croat. 55, 279-289 (2016).

103. Fernández-González, A. et al. Clinical, histological and homeostasis evaluation of an autologous tissue bio-engineered skin substitute in a patient with $70 \%$ of total body surface area (TBSA) burn. Cytotherapy 19, S233 (2017).

104. Boyce, S. T. et al. Randomized, paired-site comparison of autologous engineered skin substitutes and split-thickness skin graft for closure of extensive, fullthickness burns. J. Burn Care Res. 38, 61-70 (2017).

105. Germain, L. et al. Autologous bilayered self-assembled skin substitutes (SASSs) as permanent grafts: a case series of 14 severely burned patients indicating clinical effectiveness. Eur. Cells Mater. 36, 128-141 (2018).

106. Meuli, M. et al. A cultured autologous dermo-epidermal skin substitute for fullthickness skin defects: a phase l, open, prospective clinical trial in children. Plast. Reconstr. Surg. 144, 188-198 (2019).

107. Liu, Y. et al. Reconstruction of a tissue-engineered skin containing melanocytes. Cell Biol. Int. 31, 985-990 (2007).

108. Biedermann, T. et al. Tissue-engineered dermo-epidermal skin analogs exhibit de novo formation of a near natural neurovascular link 10 weeks after transplantation. Pediatr. Surg. Int. 30, 165-172 (2014).

109. Biedermann, T., Klar, A. S., Böttcher-Haberzeth, S., Reichmann, E. \& Meuli, M. Myelinated and unmyelinated nerve fibers reinnervate tissue-engineered dermo-epidermal human skin analogs in an in vivo model. Pediatr. Surg. Int. 32, 1183-1191 (2016).

110. Klar, A. S. et al. Differential expression of granulocyte, macrophage, and hypoxia markers during early and late wound healing stages following transplantation of tissue-engineered skin substitutes of human origin. Pediatr. Surg. Int. 30, 1257-1264 (2014).

111. Boyce, S. T. et al. Restoration of cutaneous pigmentation by transplantation to mice of isogeneic human melanocytes in dermal-epidermal engineered skin substitutes. Pigment Cell Melanoma Res. 30, 531-540 (2017).

112. Goyer, B. et al. Impact of ultraviolet radiation on dermal and epidermal DNA damage in a human pigmented bilayered skin substitute. J. Tissue Eng. Regen. Med. 13, 2300-2311 (2019).

113. Supp, D. M. et al. Light or dark pigmentation of engineered skin substitutes containing melanocytes protects against ultraviolet light-induced DNA damage in vivo. J. Burn Care Res. https://doi.org/10.1093/JBCR/IRAA029 (2020).

114. Deckers, J., Hammad, H. \& Hoste, E. Langerhans cells: sensing the environment in health and disease. Front. Immunol. 9, 93 (2018).

115. Abraham, J. \& Mathew, S. Merkel cells: a collective review of current concepts. Int. J. Appl. basic Med. Res. 9, 9-13 (2019).

116. Bechetoille, N. et al. Effects of solar ultraviolet radiation on engineered human skin equivalent containing both langerhans cells and dermal dendritic cells. Tissue Eng. 13, 2667-2679 (2007).

117. Laubach, V. et al. Integration of Langerhans-like cells into a human skin equivalent. Arch. Dermatol. Res. 303, 135-139 (2011).

118. Fransson, J., Heffler, L. C., Linder, M. T. \& Scheynius, A. Culture of human epidermal Langerhans cells in a skin equivalent. Br. J. Dermatol. 139, 598-604 (1998).

119. Régnier, M., Patwardhan, A., Scheynius, A. \& Schmidt, R. Reconstructed human epidermis composed of keratinocytes, melanocytes and Langerhans cells. Med. Biol. Eng. Comput. 36, 821-824 (1998).

120. Hafemann, B. et al. Use of a collagen/elastin-membrane for the tissue engineering of dermis. Burns 25, 373-384 (1999).

121. Centanni, J. M. et al. Stratagraft skin substitute is well-tolerated and is not acutely immunogenic in patients with traumatic wounds: Results from a prospective, randomized, controlled dose escalation trial. Ann. Surg. 253, 672-683 (2011).
122. Hahn, J. M. et al. Identification of Merkel cells associated with neurons in engineered skin substitutes after grafting to full thickness wounds. PLOS ONE 14, e0213325 (2019).

123. Ojeh, N., Pastar, I., Tomic-Canic, M. \& Stojadinovic, O. Stem cells in skin regeneration, wound healing, and their clinical applications. Int. J. Mol. Sci. 16, 16 (2015).

124. Shi, C., Zhu, Y., Su, Y. \& Cheng, T. Stem cells and their applications in skin-cell therapy. Trends Biotechnol. 24, 48-52 (2006).

125. Martin, M. T., Vulin, A. \& Hendry, J. H. Human epidermal stem cells: role in adverse skin reactions and carcinogenesis from radiation. Mutat. Res. -Rev. Mutat. Res. 770, 349-368 (2016).

126. Potten, C. S. \& Booth, C. Keratinocyte stem cells: a commentary. J. Invest. Dermatol. 119, 888-899 (2002).

127. Sellheyer, K. \& Krahl, D. Skin mesenchymal stem cells: prospects for clinical dermatology. J. Am. Acad. Dermatol. 63, 859-865 (2010).

128. Jeremias, T. et al. Dermal substitutes support the growth of human skin-derived mesenchymal stromal cells: potential tool for skin regeneration. PLOS ONE 9 , e89542 (2014).

129. Salerno, S. et al. Dermal-epidermal membrane systems by using human keratinocytes and mesenchymal stem cells isolated from dermis. Mater. Sci. Eng. C. 71, 943-953 (2017).

130. Leirós, G. J. et al. Dermal papilla cells improve the wound healing process and generate hair bud-like structures in grafted skin substitutes using hair follicle stem cells. Stem Cells Transl. Med. 3, 1209-1219 (2014).

131. Higgins, C. A. et al. Multifaceted role of hair follicle dermal cells in bioengineered skins. Br. J. Dermatol. 176, 1259-1269 (2017).

132. Mohd Hilmi, A. B., Hassan, A. \& Halim, A. S. A bilayer engineered skin substitute for wound repair in an irradiation-impeded healing model on rat. Adv. Wound Care 4, 312-320 (2015).

133. Michalak-Micka, K. et al. Impact of human mesenchymal cells of different body site origins on the maturation of dermo-epidermal skin substitutes. Pediatr. Surg. Int. 35, 121-127 (2019).

134. Zomer, H. D., Jeremias, T., da, S., Ratner, B. \& Trentin, A. G. Mesenchymal stromal cells from dermal and adipose tissues induce macrophage polarization to a prorepair phenotype and improve skin wound healing. Cytotherapy https://doi.org/ 10.1016/j.jcyt.2020.02.003 (2020).

135. Takahashi, K. et al. Induction of pluripotent stem cells from adult human fibroblasts by defined factors. Cell 131, 861-872 (2007).

136. Itoh, M. et al. Generation of 3D skin equivalents fully reconstituted from human induced pluripotent stem cells (iPSCs). PLOS ONE 8, e77673 (2013).

137. Gledhill, K. et al. Melanin transfer in human 3D skin equivalents generated exclusively from induced pluripotent stem cells. PLOS ONE 10, e0136713 (2015).

138. Petrova, A. et al. 3D in vitro model of a functional epidermal permeability barrier from human embryonic stem cells and induced pluripotent stem cells. Stem Cell Rep. 2, 675-689 (2014).

139. Kim, Y. et al. Establishment of a complex skin structure via layered co-culture of keratinocytes and fibroblasts derived from induced pluripotent stem cells. Stem Cell Res. Ther. 9, 217 (2018).

140. Muller, Q. et al. Development of an innervated tissue-engineered skin with human sensory neurons and Schwann cells differentiated from iPS cells. Acta Biomater. 82, 93-101 (2018).

141. Lin, Y. \& Hogan, W. J. Clinical application of mesenchymal stem cells in the treatment and prevention of graft-versus-host disease. Adv. Hematol. 2011, 1-17 (2011).

142. Liang, J. et al. Allogenic mesenchymal stem cells transplantation in refractory systemic lupus erythematosus: a pilot clinical study. Ann. Rheum. Dis. 69, 1423-1429 (2010)

143. Koppula, P. R., Chelluri, L. K., Polisetti, N. \& Vemuganti, G. K. Histocompatibility testing of cultivated human bone marrow stromal cells-A promising step towards pre-clinical screening for allogeneic stem cell therapy. Cell. Immunol. 259, 61-65 (2009).

144. Squillaro, T., Peluso, G. \& Galderisi, U. Clinical trials with mesenchymal stem cells: an update. Cell Transplant. 25, 829-848 (2016).

145. Ankrum, J. A., Ong, J. F. \& Karp, J. M. Mesenchymal stem cells: immune evasive, not immune privileged. Nat. Biotechnol. 32, 252-260 (2014).

146. Lohan, P., Treacy, O., Griffin, M. D., Ritter, T. \& Ryan, A. E. Anti-donor immune responses elicited by allogeneic mesenchymal stem cells and their extracellular vesicles: are we still learning? Front. Immunol. 8, 1626 (2017).

147. Galipeau, J. \& Sensébé, L. Mesenchymal stromal cells: clinical challenges and therapeutic opportunities. Cell Stem Cell 22, 824-833 (2018).

148. Di Nicola, M. et al. Human bone marrow stromal cells suppress T-lymphocyte proliferation induced by cellular or nonspecific mitogenic stimuli. Blood 99, 3838-3843 (2002) 
149. Seo, B. F., Kim, K. J., Kim, M. K. \& Rhie, J. W. The effects of human keratinocyte coculture on human adipose-derived stem cells. Int. Wound J. 13, 630-635 (2016).

150. Zuk, P. A. et al. Human adipose tissue is a source of multipotent stem cells. Mol. Biol. Cell 13, 4279-4295 (2002).

151. Ghannam, S. et al. Mesenchymal stem cells inhibit human Th17 cell differentiation and function and induce a T regulatory cell phenotype. J. Immunol. 185, 302-312 (2010).

152. Martin-Piedra, M. et al. Effective use of mesenchymal stem cells in human skin substitutes generated by tissue engineering. Eur. Cells Mater. 37, 233-249 (2019).

153. He, L. J. et al. Full-thickness tissue engineered skin constructed with autogenic bone marrow mesenchymal stem cells. Sci. China, Ser. C. Life Sci. 50, 429-437 (2007).

154. Ojeh, N. O. \& Navsaria, H. A. An in vitro skin model to study the effect of mesenchymal stem cells in wound healing and epidermal regeneration. J. Biomed. Mater. Res. 102, 2785-2792 (2014).

155. Kalaszczynska, I. \& Ferdyn, K. Wharton's jelly derived mesenchymal stem cells: future of regenerative medicine? Recent findings and clinical significance. Biomed. Res. Int. 2015, 430847 (2015).

156. Garzón, l. et al. Wharton's jelly stem cells: a novel cell source for oral mucosa and skin epithelia regeneration. Stem Cells Transl. Med. 2, 625-632 (2013).

157. Ertl, J. et al. Comparative study of regenerative effects of mesenchymal stem cells derived from placental amnion, chorion and umbilical cord on dermal wounds. Placenta 65, 37-46 (2018).

158. Shi, S., Jia, S., Liu, J. \& Chen, G. Accelerated regeneration of skin injury by cotransplantation of mesenchymal stem cells from Wharton's jelly of the human umbilical cord mixed with microparticles. Cell Biochem. Biophys. 71, 951-956 (2015).

159. Millán-Rivero, J. E. et al. Silk fibroin scaffolds seeded with Wharton's jelly mesenchymal stem cells enhance re-epithelialization and reduce formation of scar tissue after cutaneous wound healing. Stem Cell Res. Ther. 10, 126 (2019).

160. Chavez-Munoz, C. et al. Transdifferentiation of adipose-derived stem cells into keratinocyte-like cells: engineering a stratified epidermis. PLOS ONE 8, e80587 (2013).

161. Ebrahimian, T. G. et al. Cell therapy based on adipose tissue-derived stromal cells promotes physiological and pathological wound healing. Arterioscler. Thromb. Vasc. Biol. 29, 503-510 (2009).

162. Paganelli, A. et al. In vitro engineering of a skin substitute based on adiposederived stem cells. Cells Tissues Organs 207, 46-57 (2019).

163. Moriyama, M. et al. Adipose-derived stromal/stem cells improve epidermal homeostasis. Sci. Rep. 9, 18371 (2019).

164. Motamed, S. et al. Cell-based skin substitutes accelerate regeneration of extensive burn wounds in rats. Am. J. Surg. 214, 762-769 (2017).

165. Zhang, A.-J., Jiang, T., Li, Q., Jin, P.-S. \& Tan, Q. Experimental research on ADSCsNCSS in wound repair. Exp. Ther. Med. 16, 4429-4436 (2018).

166. Mendez, J. J. et al. Mesenchymal stromal cells form vascular tubes when placed in fibrin sealant and accelerate wound healing in vivo. Biomaterials 40, 61-71 (2015).

167. Huang, S.-P. et al. Adipose-derived stem cells seeded on acellular dermal matrix grafts enhance wound healing in a murine model of a full-thickness defect. Ann. Plast. Surg. 69, 656-662 (2012).

168. Sánchez-Muñoz, I. et al. The use of adipose mesenchymal stem cells and human umbilical vascular endothelial cells on a fibrin matrix for endothelialized skin substitute. Tissue Eng. 21, 214-223 (2015).

169. Klar, A. S. et al. Human adipose mesenchymal cells inhibit melanocyte differentiation and the pigmentation of human skin via increased expression of TGFß1. J. Invest. Dermatol. 137, 2560-2569 (2017).

170. Klar, A. S. et al. Tissue-engineered dermo-epidermal skin grafts prevascularized with adipose-derived cells. Biomaterials 35, 5065-5078 (2014).

171. Klar, A. S. et al. Characterization of vasculogenic potential of human adiposederived endothelial cells in a three-dimensional vascularized skin substitute. Pediatr. Surg. Int. 32, 17-27 (2016).

172. Vojtassák, J. et al. Autologous biograft and mesenchymal stem cells in treatment of the diabetic foot. Neuro Endocrinol. Lett. 27, 134-137 (2006).

173. Yoshikawa, T. et al. Wound therapy by marrow mesenchymal cell transplantation. Plast. Reconstr. Surg. 121, 860-877 (2008).

174. Xu, Y., Huang, S., Fu, X. \& Fu, X. Autologous transplantation of bone marrowderived mesenchymal stem cells: a promising therapeutic strategy for prevention of skin-graft contraction. Clin. Exp. Dermatol. 37, 497-500 (2012).

175. Arkoulis, N., Watson, S. \& Weiler-Mithoff, E. Stem cell enriched dermal substitutes for the treatment of late burn contractures in patients with major burns. Burns 44, 724-726 (2018).

176. Moon, K. C. et al. Potential of allogeneic adipose-derived stem cell-hydrogel complex for treating diabetic foot ulcers. Diabetes 68, 837-846 (2019).
177. Stessuk, T. et al. A topical cell therapy approach for diabetic chronic ulcers: effects of mesenchymal stromal cells associated with platelet-rich plasma. J. Cosmet. Dermatol. https://doi.org/10.1111/jocd.13321 (2020).

178. Hashemi, S. S. et al. The healing effect of Wharton's jelly stem cells seeded on biological scaffold in chronic skin ulcers: a randomized clinical trial. J. Cosmet. Dermatol. 18, 1961-1967 (2019).

179. Naik, S., Larsen, S. B., Cowley, C. J. \& Fuchs, E. Two to tango: dialog between immunity and stem cells in health and disease. Cell 175, 908-920 (2018).

180. Ali, N. \& Rosenblum, M. D. Regulatory T cells in skin. Immunology 152, 372-381 (2017).

181. O'Neill, L. A. J., Kishton, R. J. \& Rathmell, J. A guide to immunometabolism for immunologists. Nat. Rev. Immunol. 16, 553-565 (2016).

182. Castellana, D., Paus, R. \& Perez-Moreno, M. Macrophages contribute to the cyclic activation of adult hair follicle stem cells. PLoS Biol. 12, e1002002 (2014).

183. Shook, B., Xiao, E., Kumamoto, Y., Iwasaki, A. \& Horsley, V. CD301b+ macrophages are essential for effective skin wound healing. J. Invest. Dermatol. 136, 1885-1891 (2016).

184. Liu, Y. et al. TGF- $\beta 1$ promotes scar fibroblasts proliferation and transdifferentiation via up-regulating MicroRNA-21. Sci. Rep. 6, 32231 (2016).

185. Han, Y. M. et al. Imbalance of T-helper 17 and regulatory T cells in patients with alopecia areata. J. Dermatol. 42, 981-988 (2015).

186. Castela, E. et al. Effects of low-dose recombinant interleukin 2 to promote Tregulatory cells in alopecia areata. JAMA Dermatol. 150, 748-751 (2014).

187. Phillips, T. J. et al. The longevity of a bilayered skin substitute after application to venous ulcers. Arch. Dermatol. 138, 1079-1081 (2002).

188. Zangi, L. et al. Direct imaging of immune rejection and memory induction by allogeneic mesenchymal stromal cells. Stem Cells 27, 2865-2874 (2009).

189. Dixit, S. et al. Immunological challenges associated with artificial skin grafts: Available solutions and stem cells in future design of synthetic skin. J. Biol. Eng. 11, 49 (2017).

190. Chattopadhyay, S. \& Raines, R. T. Review collagen-based biomaterials for wound healing. Biopolymers 101, 821-833 (2014).

191. Bi, Y., Patra, P. \& Faezipour, M. Structure of collagen-glycosaminoglycan matrix and the influence to its integrity and stability. in 2014 36th Annual International Conference of the IEEE Engineering in Medicine and Biology Society, EMBC 2014. Vol. 2014, 3949-3952 (Institute of Electrical and Electronics Engineers Inc., 2014).

192. Lee, D. H., Oh, J. H. \& Chung, J. H. Glycosaminoglycan and proteoglycan in skin aging. J. Dermatological Sci. 83, 174-181 (2016).

193. Fallacara, A., Manfredini, S., Durini, E. \& Vertuani, S. Hyaluronic acid fillers in soft tissue regeneration. Facial Plast. Surg. 33, 087-096 (2017).

194. Yang, J.-A. et al. Transdermal delivery of hyaluronic acid-Human growth hormone conjugate. Biomaterials 33, 5947-5954 (2012).

195. Greene, J. J. \& Sidle, D. M. The hyaluronic acid fillers. Facial Plast. Surg. Clin. North Am. 23, 423-432 (2015).

196. Nicoletti, G. et al. Long-term in vivo assessment of bioengineered skin substitutes: a clinical study. J. Tissue Eng. Regen. Med. 9, 460-468 (2015).

197. Nicoletti, G. et al. Versatile use of dermal substitutes: a retrospective survey of 127 consecutive cases. Indian J. Plast. Surg. 51, 46-53 (2018).

198. Chen, W. Y. \& Abatangelo, G. Functions of hyaluronan in wound repair. Wound Repair Regen. 7, 79-89 (1999).

199. Alexaline, M. M. et al. Influence of fibrin matrices and their released factors on epidermal substitute phenotype and engraftment. J. Tissue Eng. Regen. Med. 13, 1362-1374 (2019).

200. Lo, V. \& Pope, E. Amniotic membrane use in dermatology. Int. J. Dermatol. 48, 935-940 (2009).

201. Sullivan, T. P., Eaglstein, W. H., Davis, S. C. \& Mertz, P. The pig as a model for human wound healing. Wound Repair Regen. 9, 66-76 (2001).

202. Debeer, S. et al. Comparative histology and immunohistochemistry of porcine versus human skin. Eur. J. Dermatol. 23, 456-466 (2013).

203. Stricker-Krongrad, A., Shoemake, C. R. \& Bouchard, G. F. The miniature swine as a model in experimental and translational medicine. Toxicol. Pathol. 44, 612-623 (2016).

204. Khiao In, M. et al. Histological and functional comparisons of four anatomical regions of porcine skin with human abdominal skin. Anat. Histol. Embryol. 48, 207-217 (2019).

205. Amini-Nik, S. et al. Stem cells derived from burned skin-The future of burn care. EBioMedicine 37, 509-520 (2018).

206. Dearman, B. L., Stefani, K., Li, A. \& Greenwood, J. E. Take of a polymer-based autologous cultured composite skin on an integrated temporizing dermal matrix: proof of concept. J. Burn Care Res. 34, 151-160 (2013).

207. Fleischmann, T., Nicholls, F., Lipiski, M., Arras, M. \& Cesarovic, N. in Methods in Molecular Biology. Vol. 1993, 251-259 (Humana Press Inc., 2019).

208. Pham, C., Greenwood, J., Cleland, H., Woodruff, P. \& Maddern, G. Bioengineered skin substitutes for the management of burns: a systematic review. Burns 33, 946-957 (2007). 
209. Greenwood, J. E. The evolution of acute burn care - retiring the split skin graft. Ann. R. Coll. Surg. Engl. 99, 432-438 (2017).

210. Back, C., Dearman, B., Li, A., Neild, T. \& Greenwood, J. E. Noncultured keratinocyte/melanocyte cosuspension: Effect on reepithelialization and repigmentation-A randomized, placebo-controlled study. J. Burn Care Res. 30, 408-416 (2009).

211. Ishack, S. \& Lipner, S. R. A review of 3-dimensional skin bioprinting techniques: applications, approaches, and trends. Dermatol. Surg. 46, 1500-1505 (2020).

212. Donnelly, H., Salmeron-Sanchez, M. \& Dalby, M. J. Designing stem cell niches for differentiation and self-renewal. J. R. Soc. Interface 15, 20180388 (2018).

\section{ACKNOWLEDGEMENTS}

This study has been funded by Instituto de Salud Carlos III through the project PI13-02576 (co-funded by European Regional Development Fund "A way to make Europe") and Regional Government of Andalusia (SAS PI-0458-2016 and salud2016-73581-tra). The work of Álvaro Sierra-Sánchez was supported by a predoctoral fellowship (BOE 05/01/2018) funded by Instituto de Salud Carlos III (co-funded by European Social Fund "Investing in your future") with the dossier number FI18/00269. This study is part of his doctoral research in the Biomedicine's program of University of Granada.

\section{AUTHOR CONTRIBUTIONS}

Á.S.-S. and K.H.K. reviewed bibliography and wrote the manuscript, G.B.-M. and S.A.-S. reviewed bibliography and revised the manuscript. Á.S.-S. and K.H.K. are co-first authors of this manuscript.

\section{COMPETING INTERESTS}

The authors declare no competing interests.

\section{ADDITIONAL INFORMATION}

Correspondence and requests for materials should be addressed to Á.S-S.

Reprints and permission information is available at http://www.nature.com/ reprints

Publisher's note Springer Nature remains neutral with regard to jurisdictional claims in published maps and institutional affiliations.

(i) Open Access This article is licensed under a Creative Commons Attribution 4.0 International License, which permits use, sharing, adaptation, distribution and reproduction in any medium or format, as long as you give appropriate credit to the original author(s) and the source, provide a link to the Creative Commons license, and indicate if changes were made. The images or other third party material in this article are included in the article's Creative Commons license, unless indicated otherwise in a credit line to the material. If material is not included in the article's Creative Commons license and your intended use is not permitted by statutory regulation or exceeds the permitted use, you will need to obtain permission directly from the copyright holder. To view a copy of this license, visit http://creativecommons. org/licenses/by/4.0/.

(c) The Author(s) 2021 